\title{
Recent Progress in Networked Control Systems - A Survey
}

\author{
Yuan-Qing Xia Yu-Long Gao Li-Ping Yan Meng-Yin Fu \\ School of Automation, Beijing Institute of Technology, Beijing 100081, China
}

\begin{abstract}
For the past decades, networked control systems (NCSs), as an interdisciplinary subject, have been one of the main research highlights and many fruitful results from different aspects have been achieved. With these growing research trends, it is significant to consolidate the latest knowledge and information to keep up with the research needs. In this paper, the results of different aspects of NCSs, such as quantization, estimation, fault detection and networked predictive control, are summarized. In addition, with the development of cloud technique, cloud control systems are proposed for the further development of NCSs.
\end{abstract}

Keywords: Networked control systems, quantization, filter, data fusion, fault detection, networked predictive control, cloud control systems.

\section{Introduction}

For the past decades, as a research highlight, networked control systems (NCSs) not only have attracted the attention of many scholars but also have generated huge profits in the industry. Compared with the traditional control systems, NCSs have obvious advantages: low cost, flexibility, easy re-configurability, natural reliability, robustness to failure, and adaptation capability. Up to now, networked control techniques have been applied into many fields, such as transportation networks, power grids, water distribution networks, telephone networks, global financial network, genetic expression networks, etc.

In general, the research of NCSs can be categorized into two parts: control of network and control over network. The former is to study the problem about network, such as routing control, congestion reduction, efficient data communication, networking protocol, etc. The latter is to achieve the performance of the control system with network being the transmission media. In this survey, we mainly discuss the latter. With respect to control over network, sharednetwork control systems and remote control systems are two important types of NCSs. The research of NCSs is focused on two aspects: the quality of service (QoS) and the quality of control (QoC). QoS is related to the performance of the network, such as transmission rates and error rates. QoC generally refers to the stability of the system. It is one of

\section{Survey Paper}

Manuscript received October 1, 2014; accepted January 26, 2015

This work was supported by National Basic Research Program of China (973 Program) (No. 2012CB720000), National Natural Science Foundation of China (Nos. 61225015 and 60974011), Foundation for Innovative Research Groups of the National Natural Science Foundation of China (No.61321002), Beijing Municipal Natural Science Foundation (Nos. 4102053 and 4101001), Beijing Natural Science Foundation (Nos. 4132042) and Beijing Higher Education Young Elite Teacher Project (No. YETP1212).

Recommended by Editor-in-Chief Guo-Ping Liu

(C) Institute of Automation, Chinese Academy of Science and Springer-Verlag Berlin Heidelberg 2015 the most important issues to maintain QoS and QoC at the same time in the research of NCSs.

Due to the existence of the network in NCSs, some communication problems may degrade the performance of the system. They are presented as follows:

1) Network-induced delay: The network-induced delay can be formulated as

$$
\tau\left(t_{k}\right)=\tau_{s c}\left(t_{k}\right)+\tau_{c a}\left(t_{k}\right)
$$

where $\tau\left(t_{k}\right)$ is the total transmission delay at sampling time $t_{k}, \tau_{s c}\left(t_{k}\right)$ and $\tau_{c a}\left(t_{k}\right)$ are the network delays from the sensor to the controller and from the controller to the actuator nodes, respectively. For NCSs, there were other delays such as $\tau_{s}\left(t_{k}\right), \tau_{c}\left(t_{k}\right)$ and $\tau_{a}\left(t_{k}\right)^{[1]}$, which are the computational delays in the sensor, controller, and actuator nodes, respectively. In the existing literatures, different delay models are introduced. In [2], four major delay models, namely constant delay model, mutually independent stochastic delay model, Markov chain model, and hidden Markov model, were summarized.

2) Packet dropout: Due to several factors such as data traffic congestion, data collision or interference, packet loss is an inherent problem in most communication networks. Generally, the dropout process is often modeled as a Bernoulli process or a Markov process.

3) Limited capacity of channel: The limited capacity of communication channel refers to the limited bandwidth and the limited transmission energy, which both influence the transmission quality.

4) Network security: Any network medium, particularly wireless medium, is susceptible to easy intercepting. Network security is receiving more and more concern.

Generally speaking, the research of NCSs is mainly to solve the aforementioned problems. Zhang et al. ${ }^{[3]}$ surveyed the main methodologies which can cope with those typical network-induced constraints, namely time delays, packet losses and disorder, time-varying transmission intervals, 


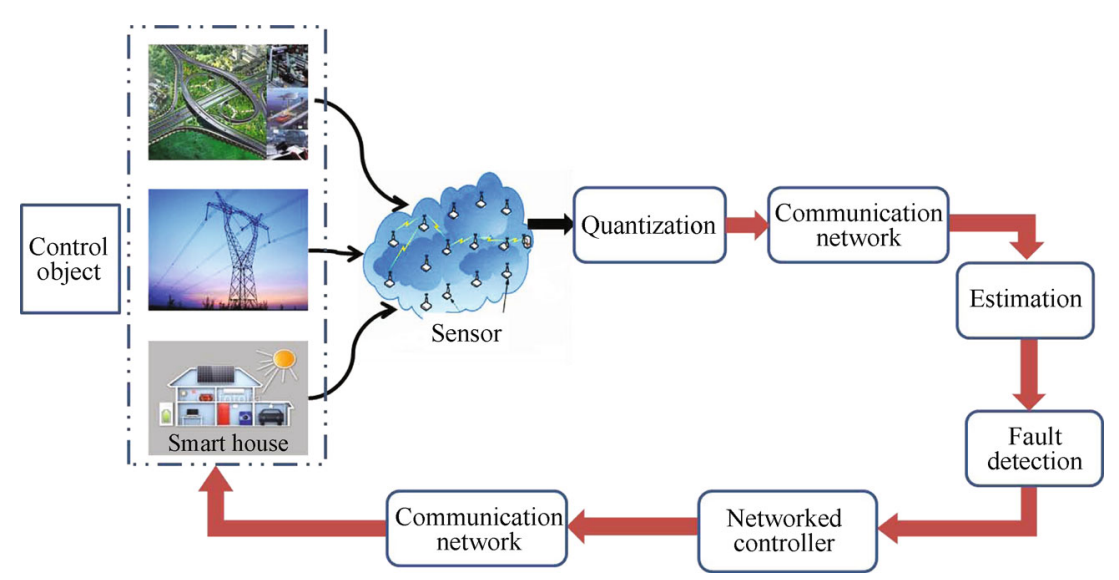

Fig. 1 The framework of NCS

competition of multiple nodes accessing networks, and data quantization. From the framework of a general NCS shown in Fig. 1, the network-induced problems may influence almost every stage. The whole closed-loop can be described as follows: The sensors of the system sense the states or the outputs. Due to the uncertainty or the disturbance, these measurements are transmitted through the communication network to estimator, such as filter or data fusion, to estimate the exact states. Then, these processed data are used to detect the fault or/and compute the control input. Finally, the control input is fed to the actual control object.

Under the effect of the network, most of the existing results about NCSs can consider only a single stage in Fig. 1. With respect to the theoretical side, many important improvements and advances have been obtained in the past decades. The motivation of this survey is to summarize these important results about NCSs and present a framework of NCSs to newcomers in this field.

The remainder of this paper is organized as follows. Section 2 presents the results about the quantization of NCSs, specially the logarithmic quantizer and the uniform quantizer. In Section 3, the estimation stage in NCSs, mainly filtering and data fusion, is summarized. Then, the results about the fault detection for NCSs are simply shown in Section 4. In Section 5, since networked predictive control is a significant approach to NCSs, the corresponding results about predictive control are categorized into model predictive control (MPC), packet based predictive control and data driven predictive control. Moreover, the distributed predictive control is also summarized in this section. Section 6 proposes the cloud control system as one of the further developments of NCSs and describes its basic idea. Conclusions are given in Section 7 .

\section{Quantization}

Quantization is an significant problem in NCSs. Owing to the limited transmission capacity of the network, signals must be quantized before they are sent to the network. Quantization is implemented by a quantizer, which is a device to convert a real-valued signal into a piecewise constant one with a finite set of values. When a network exists in the system, quantization is motivated by the communication constraints. To achieve better performance of the considered systems, the effect of data quantization on the system should be taken into consideration.

A continuous variable is converted into a discretized variable by quantizer. Generally, this will inevitably introduce information loss due to the existence of quantization error. A fundamental problem in NCSs is how the quantization error will affect the quality of the system operation. It is easy to see that the less number of bits we use for quantization, the larger quantization error will be induced. If the quantization error is too large, the controller may not be able to generate a stabilizing control input. Thus, one branch of the study of the quantization is focused on how to design a proper quantizer and achieve the stability of the system.

The existence of the quantizer will result in two phenomenons, i.e., saturation and performance deterioration around the original point. If the signal exceeds the quantization range of the quantizer, a large quantization error may occur and result in that the closed-loop system is unstable. This is the influence of the saturation. If the state approaches to the original point, the limitation of the quantizer's accuracy will lead to that the signal cannot be exactly quantized. In this case, the closed-loop system inevitably cannot achieve the asymptotic stability.

The early work on quantized feedback control is mainly motivated by digital computers as instrument for implementing control systems. While the network is introduced as the transmission medium, new techniques need to be studied. In [4], it is pointed out that when ordinary "linear" feedback of quantized state measurements is applied, the closed-loop system will behave chaotically. When the state is one-dimensional, a quantitative statistical analysis of the resulting closed-loop dynamics reveals that the existence of an invariant probability measure on the state space, which is absolutely continuous with respect to the Lebesgue measure and with respect to which the closed-loop system is ergodic. In addition, as a hot topic, the event-triggering control with quantization was explored ${ }^{[5-8]}, \mathrm{Hu}$ and Yue ${ }^{[6]}$ 
considered both state and control input quantization and proposed an asymptotical stability criteria in terms of linear matrix inequalities (LMIs).

Nowadays, the logarithmic quantizer and the uniform quantizer are two popular quantizers. When one compares the logarithmic quantizer with the uniform one, it is not hard to obtain that the former yields better performance around the origin and the latter can be operated more easily. Each kind of quantizer has both advantages and disadvantages. In the following, we will discuss the logarithmic quantizer and the uniform quantizer, respectively.

\subsection{Logarithmic quantizer}

The logarithmic quantizer is a kind of the static quantizer. It is formulated as

$$
q(y)=\left\{\begin{array}{l}
v_{i}, \text { if } \frac{v_{i}}{1+\delta}<y<\frac{v_{i}}{1-\delta}, y>0 \\
0, \text { if } y=0 \\
-q(-y), \text { if } y<0
\end{array}\right.
$$

where $U=\left\{ \pm v_{i}: v_{i}=\rho^{i} v_{0}, i= \pm 1, \pm 2, \cdots\right\} \cup\left\{ \pm v_{0}\right\} \cup$ $\{0\}, 0<\rho<1, v_{0}>0$ is the set of the quantization values. This quantizer is of infinite quantization level. A quantizer with limited quantitative level is

$$
q(y)=\left\{\begin{array}{l}
v_{i}, \text { if } \frac{v_{i}}{1+\delta}<y<\frac{v_{i}}{1-\delta}, 0<i \leq N-1 \\
0, \text { if } 0 \leq y \leq \frac{v_{N-1}}{1+\delta} \\
v_{0}, \text { if } y>\frac{v_{0}}{1+\delta} \\
-q(-y), \text { if } y<0
\end{array}\right.
$$

where $U=\left\{ \pm v_{i}: v_{i}=\rho^{i} v_{0}, i= \pm 1, \pm 2, \cdots, \pm(N-1)\right\} \cup$ $\left\{ \pm v_{0}\right\} \cup\{0\}, 0<\rho<1, v_{0}>0$ is the set of the quantization values. Fig. 2 shows an example of the logarithmic quantizer.

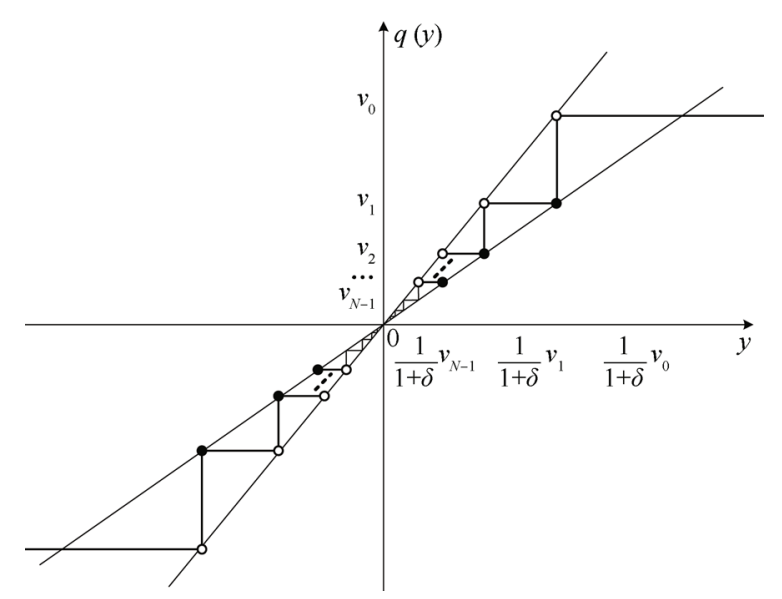

Fig. 2 An example of logarithmic quantizer

The logarithmic quantizer was firstly proposed in [9]. Elia and Mitter ${ }^{[9]}$ turned out that the logarithmic quantizer is the coarsest quantizer which has the minimum quantization density. This work also discussed the quadratic stabi- lization of single-input linear systems with quantized control feedback. Fu and $\mathrm{Xie}^{[10]}$ extended the results of [9] to multiple input multiple output (MIMO) systems and presented that the quantization feedback design problem can be converted into the robust control problem. This is crucial for the research of the logarithmic quantizer. In [11], quantization issues were studied in a remote control system. Hayakama et al. ${ }^{[12,13]}$ studied the adaptive quantized control for the linear uncertain discrete-time systems and nonlinear uncertain systems, respectively. You et al. ${ }^{[14]}$ investigated the attainability of the minimum average data rate for stabilization of linear systems via logarithmic quantization. By exploring some geometric properties of the logarithmic quantizer and using the fact that the logarithmic quantizer is sector bounded and nondecreasing, Zhou et al. ${ }^{[15]}$ presented a new approach to the stability analysis of quantized feedback control systems.

For NCSs, the time delay and the packet loss are two usual issues. Due to their existence, most of the traditional logarithmic quantizers are not suitable. Up to now, some quantization results for the NCSs have been obtained. In [16], the guaranteed cost control design for linear systems with parameter uncertainty was considered. The logarithmic quantizers were used in two sides and the effects of both the quantization levels and the network conditions are also considered. Zhang et al. ${ }^{[17,18]}$ discussed a class of $H_{2}$ filters and $H_{\infty}$ filters for nonlinear systems with logarithmic quantizer and packet loss, respectively. Li et al. ${ }^{[19]}$ considered the robust $H_{\infty}$ control problem for uncertain discretetime Takagi and Sugeno (T-S) fuzzy networked control systems with state quantization, where a logarithmic quantizer was used and new model of network-based control with simultaneous consideration of network-induced delays and packet dropouts was constructed. A less conservative delaydependent stability condition was thus derived. In [20], the input quantization and packet dropouts were considered simultaneously. The packet dropout process of the channel was modelled as an independent identically distributed case and a logarithmic quantizer was adopted. It turns out that as long as the dropout rate satisfies $\alpha<1+\frac{\ln \Lambda}{\ln \delta-l \ln (1+\delta)}$, the system is globally exponentially stabile, i.e.,

$$
\mathrm{E}\left(\left[\|x(t)-\widetilde{x}(t)\|_{\infty}\right]\right)<\mathrm{e}^{-\lambda t} X
$$

where $t>0$, E represents the mean, $\Lambda=\mathrm{e}^{F T}, T$ is the sample interval, $F$ is one positive real number, $\delta>\rho^{N-1}$, $N \geq 3,0<\rho<1,\|x(0)\|_{\infty}<X, \lambda=\frac{\ln R}{T}$ and $R=\Lambda\left(\frac{\delta}{1+\delta}\right)^{1-\alpha}<1$. Xia et al. ${ }^{[21]}$ considered a quantized system with finite-level quantized input computed from quantized measurements (QIQM). The problem of globally asymptotic stability of QIQM system is converted into the one of an equivalent system depending on a multiplier which is nonnegative and bounded. If the open-loop system is stable and the closed transfer function satisfies 
that $\inf _{w \in \mathbf{R}} \operatorname{Re}\left\{G\left(\mathrm{e}^{\mathrm{j} w}\right)\right\}>\varepsilon$, where

$$
\begin{gathered}
q(y)=\left\{\begin{array}{c}
\frac{1}{(\delta-1)(\delta+1)^{2}} \Lambda, \text { if } 0<\delta \leq \frac{1}{2} \\
\frac{(2 \delta-1) \delta}{(1-\delta)^{2}(\delta+1)^{2}} \Lambda+\frac{1}{(\delta-1)(\delta+1)^{2}} \Lambda, \\
\text { if } \frac{1}{2} \leq \delta<1
\end{array}\right. \\
\Lambda=\frac{F_{q}}{Q\left(F_{q}\right)}-\frac{\delta\left\|F_{q}\right\|_{1}}{Q\left(F_{q}\right)} \\
F_{q}=\inf \tilde{F}=\inf \left\{F \eta \mid \eta \in Z_{\eta}= \pm \rho^{i} v_{0}, i \in\{0, i, \cdots, N\},\right. \\
\left.F \eta \geq \frac{\rho+1}{2 \rho} v_{0}\right\}
\end{gathered}
$$

and $F$ is the feedback matrix, $Q$ is the quantizer, $\rho>1$ and $v_{0}>0$, then the closed-loop system is globally asymptotically stable about origin.

As we can see, the existing results involving logarithmic quantizer are mainly related to the linear systems with infinite quantization levels. Then, how to analyze the stability of QIQM nonlinear systems, which are affected by finite logarithmic quantization levels, is a hard problem to be solved. Moreover, the problems mentioned above, which are affected by other networked induced issues such as timedelay and packet dropout simultaneously, are beyond doubt the focal and difficult points of future study.

\subsection{Uniform quantizer}

The uniform quantizers with an arbitrarily shaped quantitative area have some following conditions:

1) if $\|y\|_{2} \leq M \mu$, then $\|q(y)-y\|_{2} \leq \Delta \mu$

2) if $\|y\|_{2}>M \mu$, then $\|q(y)\|_{2}>M \mu-\Delta \mu$

where $M$ is the saturation value and $\Delta$ is the sensitivity. The first condition gives the upper bound of the quantization error when the quantization is not saturated. The second condition provides an approach to test whether the quantization is saturated. Specially, as shown in Fig. 3, an uniform quantizer whose quantitative area can be rectangle is formulated as

$q(y)=\left\{\begin{array}{l}0, \text { if }-\frac{1}{2}<y<\frac{1}{2} \\ i, \text { if } \frac{2 i-1}{2}<y<\frac{2 i+1}{2}, \quad i=1,2, \cdots, K-1 \\ K, \text { if } y \geq \frac{2 K-1}{2} \\ -q(-y), \text { if } y \leq-\frac{1}{2} .\end{array}\right.$

When a uniform quantizer is used, the zoom strategy is a useful control policy, which is divided into two stages: "zoom-in" and "zoom-out". If the initial state to be quantized is saturated, "zoom-out" stage is adopted to increase the sensitivity $\Delta$ until the state gets unsaturated. In the "zoom-in" stage, the sensitivity $\Delta$ is decreased to drive the state to zero. Otherwise, if the initial state to be quantized is unsaturated, the "zoom-out" stage can be omitted and the "zoom-out" stage is implemented directly.

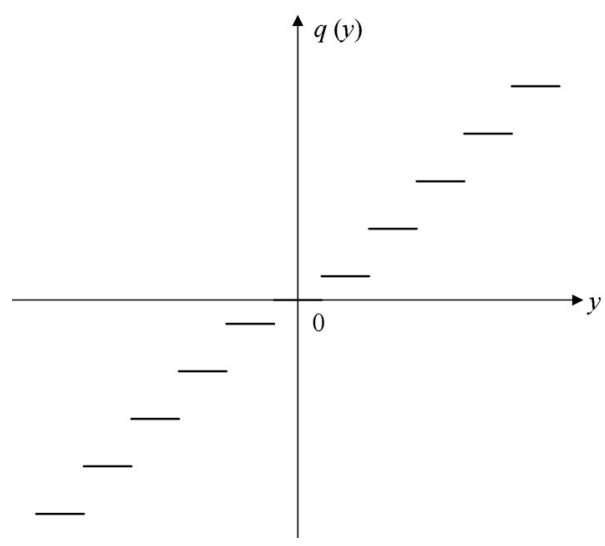

Fig. 3 An example of uniform quantizer

Brokett et al. ${ }^{[22,23]}$ proposed the uniform quantizers with an arbitrarily shaped quantitative area. Based on these quantizers, the zoom theory is used for linear systems, and the nonlinear systems, and the sufficient condition for the asymptotical stability is given. The stabilization problem for discrete time linear systems with multidimensional state and one-dimensional input using quantized feedbacks with a memory structure was investigated in [24]. Bullo and Liberzon $^{[25]}$ discussed the local optimization of the quantized control. In order to obtain a control strategy which yields arbitrarily small values of $\frac{T}{\ln C}$ (a weak form of the pole assignability property), $\frac{L N}{\ln C}$ has to be big enough, where $T$ is the time that shrinks the state of the plant from a starting set to a target set, $C$ is the contraction rate, $L$ is the number of the controller states and $N$ denotes the number of the possible control values. Reference [26] is the extension of the previous results. Reference [27] presented a general framework of the design and analysis approach for the quantized systems.

Meanwhile, considering the delay and the packet loss which the network induces, some improvements for the uniform quantizer have been obtained. For the linear discretetime system with quantization and packet dropout, You and $\mathrm{Xie}^{[28,29]}$ proposed the necessary and a sufficient condition for the mean square stability through quantization feedback. The dropout process of [28] is a Bernoulli process and the dropout process of [29] is a Markov chain. However, in these works, the relationship between the dropout rate and the eigenvalues of the system matrix influences the performance of the quantized system. The quantitative area of the uniform quantizer is a rectangle. Using uniform quantizer, Tian et al. ${ }^{[30]}$ explored quantised stabilisation and quantised $H_{\infty}$ control design for NCSs. The effects of both network-induced delay and quantization levels were considered. The design was shown in terms of LMIs. For the nonlinear system with quantization, Persis ${ }^{[31]}$ turned out that if the nonlinear system can be stabilised by "standard" state feedback, then it can be stabilised by encoded state feedback. In [32], considering data rate constraints, Persis studied the problem of stabilisation of nonlinear systems using output measurements, and an "embedded-observer" decoder and a controller were designed to guarantee the ro- 
bust practical stability of the system. Yan et al. ${ }^{[33]}$ considered the mean square stability based on the zoom strategy and Lyapunov theory. Given a fixed packet dropout rate, the sufficient condition for the stability of the closed-loop system was given in the form of LMI. It is independent of the eigenvalues of the system matrix. Moreover, the quantizer is of arbitrarily shaped quantitative area. When the dropout process is a Markov process for the given failure rate and recovery rate, sufficient conditions were given for the closed-loop fuzzy systems to be mean square stable, and the feedback controllers were designed to ensure the mean square stabilities of fuzzy systems ${ }^{[34]}$. Xia et al. ${ }^{[35]}$ explored the design and stability analysis of networked control systems with quantization and noise. Kalman filter $(\mathrm{KF})$ is used to obtain the estimated states and the quantization regions of uniform quantizer were of rectangular shapes. Based on Lyapunov theory and invariable set theory, a sufficient condition for the stability of the closedloop system was given. Under the effect of the quantization and the unknown disturbance, for the linear system, Sharon and Liberzon ${ }^{[36]}$ studied the input-to-state stability of the closed-loop system. The proposed controller switches repeatedly between "zooming-out" and "zooming-in". Two modes to implement the "zooming-in" phases, which attenuate an unknown disturbance while using the minimal number of quantization regions, were designed.

Similar to logarithmic quantizer, the problem of stability of nonlinear systems, especially for QIQM ones, which are affected by uniform quantizer with zoom stagey, time-delay and packet dropout, is the main and hard direction in the future study. Moreover, the quantized value of zoom stagey in each step is undertrained. Therefore, it may be the interesting problem to combine the zoom stagey and logarithmic quantizer to confirm each step quantized value to improve the closed-loop system performance.

\section{$3 \quad$ Filter and data fusion}

Due to the complexity of the network, state estimation has become a crucial and significant research field in NCSs. In general, there are two kinds of approaches for state estimation: filtering and data fusion. A filter is usually used to estimate the states of the system with measurement noise. Data fusion is the technique in which data from multiple sensors and related information are combined to improve accuracy. Mostly, many scholars use filter-based fusion to get a more accurate state estimation. In the following, we will discuss the filtering and data fusion in NCSs, respectively.

\subsection{Filtering}

The filter is to estimate the states or a linear combination of them by using the measured system inputs and outputs. KF was first proposed by Kalman ${ }^{[37]}$ in 1960 and it is mainly used for the linear systems. KF is a kind of optimal filter and can be easily and properly implemented by computer. Some modified KFs are proposed to improve the performance of the system. For example, in order to reduce the number of measurements to be transmitted from sensor to estimator, the design of transmission scheduler and estimator for linear discrete-time stochastic systems was considered ${ }^{[38]}$. However, NCSs are mostly nonlinear systems. Filters, which are fit for nonlinear systems, are extremely desired. In general, there are two kinds of methods when designing the filtering for nonlinear dynamic systems. One is to linearize the nonlinear systems. The extended Kalman filter (EKF) proposed by Sunahara and Bucy was motivated by this thought. The other one is to adopt the sample of nonlinear distribution. Typical filters are the particle filter and the unscented Kalman filter (UKF) based on unscented transformation (UT). Considering the characters of the NCSs such as the delay, the packet dropout and the channel attenuation, the filtering for NCSs is researched by many scholars. In the following, we will present the recent research results of the filtering for linear systems and nonlinear systems, respectively.

\subsubsection{Filtering for linear systems}

Up to now, there are numerous filtering approaches for linear systems. In general, the continuous-time linear system is formulated as

$$
\begin{aligned}
& \dot{x}(t)=A_{c}(t) x(t)+w(t) \\
& z(t)=C_{c}(t) x(t)+v(t)
\end{aligned}
$$

and the discrete-time vision is

$$
\begin{aligned}
& x(k+1)=A_{d}(k) x(k)+w(k) \\
& z(k)=C_{d}(k) x(k)+v(k)
\end{aligned}
$$

where $x$ is the state, $z$ is the measurement, $A_{c}$ or $A_{d}$ is the system matrix, and $C_{c}$ or $C_{d}$ is the output matrix, $w$ is the process noise, and $v$ is the measurement noise.

In the last few years, filtering with random packet dropouts in NCSs was the focus of several research studies ${ }^{[39-41]}$. As in [40], the $H_{\infty}$ filtering for NCSs with the packet loss is of the following form

$$
\begin{aligned}
& \hat{x}(k+1)=a_{f} \hat{x}(k)+b_{f} u(k)+c_{f} y(k) \\
& \hat{z}(k)=L_{f} \hat{x}(k)
\end{aligned}
$$

where $\hat{x}(k+1)$ is the estimate of the state, $u(k)$ is the system input, $y(k)$ is the output, $\hat{z}_{t}$ is the estimated signal, and $a_{f}, b_{f}, c_{f}, L_{f}$ are the filter parameters to be designed. A set of linear matrix inequalities is used to solve the corresponding optimal filter design problem, which is convex. Zhang et al. ${ }^{[4]}$ studied the $H_{\infty}$ filtering problem for networked discrete-time general multipleinput-multiple-output (MIMO) system with random packet losses. Each measurement loss process is described by a two-state Markov chain. The desired filters with minimized $H_{\infty}$ noise attenuation level bound is designed by solving a convex optimization problem. The presented necessary and sufficient condition describes a relation between the packet loss probability and two parameters (the exponential decay rate of the filtering error system and the $H_{\infty}$ noise attenuation level), to make the system mean-square exponentially 
stable and achieve a prescribed $H_{\infty}$ noise attenuation performance. Sinopoli et al. ${ }^{[42]}$ considered the discrete-time linear time-invariant system similar to (7) and designed the modified $\mathrm{KF}$ with the random arrival:

$$
\begin{aligned}
& \hat{x}(k+1 \mid k)=A \hat{x}(k \mid k) \\
& P(k+1 \mid k)=A P(k \mid k) A^{\mathrm{T}}+Q \\
& \hat{x}(k+1 \mid k+1)=\hat{x}(k+1 \mid k)+P(k+1 \mid k) C^{\mathrm{T}} \times \\
& \left(C P(k+1 \mid k) C^{\mathrm{T}}+\gamma(k+1) R+(1-\gamma(k+1)) \times\right. \\
& \left.\sigma^{2} I\right)^{-1} \times(z(k+1)-C \hat{x}(k+1 \mid k)) \\
& P(k+1 \mid k+1)=P(k+1 \mid k)-P(k+1 \mid k) C^{\mathrm{T}} \times \\
& \left(C P(k+1 \mid k) C^{\mathrm{T}}+\gamma(k+1) R+(1-\gamma(k+1))\right. \\
& \left.\sigma^{2} I\right)^{-1} \times C P(k+1 \mid k)
\end{aligned}
$$

where $Q$ and $R$ are the covariance matrices of $w$ and $v$, $\gamma(k)$ is the drop loss rate, $\sigma^{2} I$ is the variance of the observation if the packet is loss. And in addition to the special instructions, $\mathrm{T}$ represents the transposition of a matrix in the survey. Taking the limit as $\sigma \rightarrow \infty$, the update equations can be obtained as

$$
\begin{gathered}
\hat{x}(k+1 \mid k+1)=\hat{x}(k+1 \mid k)+\gamma(k+1) \times \\
K(k+1)(z(k+1)-C \hat{x}(k+1 \mid k)) \\
P(k+1 \mid k+1)=P(k+1 \mid k)-\gamma(k+1) \times \\
K(k+1) C P(k+1 \mid k)
\end{gathered}
$$

where $K(k+1)=P(k+1 \mid k) C^{\mathrm{T}}\left(C P(k+1 \mid k) C^{\mathrm{T}}+R\right)^{-1}$ is the filtering gain matrix and $\gamma_{k}$ is a random binary variable which represents that whether the observation arrives.

The state delay is another issue when designing the filter. He et al. ${ }^{[43]}$ designed a new robust $H_{\infty}$ filter for a class of networked systems with multiple state-delays. Certain LMIs, which depend on the occurrence probability of both the random sensor delay and missing measurement, are established to ensure the existence of the desired filters. $\mathrm{Hu}$ and Yue ${ }^{[44]}$ explored event-based $H_{\infty}$ filtering for networked systems with communication delay. The sufficient conditions for the exponential stability was given in terms of LMIs.

Mostly, many other results considered both the packed dropout and the state delay at the same time ${ }^{[42,45-49]}$. In [45], the adaptive filtering schemes were proposed for state estimation in NCSs with mixed uncertainties of random measurement delays, packet dropouts and missing measurements. The filter gains can be obtained by solving a set of recursive discrete-time Riccati equations. In [46], for linear systems with both random delay and packet drop, Schenato presented two alternative estimator architectures which are more computationally efficient, and provided upper and lower bounds for the performance of the time-varying estimator. The stability of these estimators does not depend on packet delay but only on the overall packet loss probability. In [47], the problem of optimal filtering of discrete-time systems with random sensor delay, multiple packet dropout and uncertain observation was investigated. The stochastic $\mathrm{H}_{2}$-norm of the estimation error was used as a criterion for the filter design and the filter was designed by solving a set of LMIs. Reference [48] is concerned with the filtering problem for a class of discrete-time stochastic nonlinear networked control systems with network-induced incomplete measurements (the multiple random communication delays and random packet losses). By using the LMI method and delay-dependent technique, sufficient conditions are derived for the exponentially mean-square stability of the filteringerror dynamics. Shi and Fang ${ }^{[4]}$ considered the problem of parameter estimation and output estimation for systems in a transmission control protocol (TCP). The input and output missing data is modeled as two separate Bernoulli processes characterised by probabilities of missing data, and a recursive algorithm for parameter estimation by modifying the KF-based algorithm is developed.

From the existing results above, we have that the filtering problem is often formulated by probabilistic approaches based on the probabilities of the uncertainties occurring between the sensor and the filter. However, a nonprobabilistic approach by time-stamping the measurement packets was proposed in [50]. The optimal state estimator was dependent on the possible control input and this approach was suitable for single-measurement packets, multiple measurement packets, the case of burst arrivals (where more than one packet may arrive between the receivers previous and current sampling times), and the scenario where the control input is non-zero and subject to delays and packet dropouts. Yue and $\operatorname{Han}^{[51]}$ not only considered the transmission delay and the packet dropout, but also studied the parameter uncertainty. The proposed criteria for $H_{\infty}$ performance analysis of the filtering-error system are expressed as a set of linear matrix inequalities. The filter design can be obtained by using convex optimization method. Similarly, Xia and Han ${ }^{[52]}$ also considered the system uncertainty and gave the sufficient condition for the design of the robust KF. Yang et al. ${ }^{[53]}$ considered a delta operator $\mathrm{KF}$ and applied it to an inverted pendulum model in experiment. A new sufficient condition of convergence analysis for the designed delta operator KF based on Lyapunov functional was given. In [54], a data-driven subspace identification method combined with the Kalman on-line filtering algorithm was proposed to solve the state estimation problem for a class of dynamical systems where the exact models can not be established. In addition, considering constrained communication energy and bandwidth, an event-based sensor data scheduler for linear systems was designed in [55] and the corresponding minimum squared error estimator was also derived.

In the following, we will introduce some filtering applications in nonlinear NCSs.

\subsubsection{Filtering for nonlinear systems}

For nonlinear systems, the stability analysis of nonlinear filter with random packet dropouts remains open. Up to now, the dropouts have been modeled as the Bernoulli 
process $^{[42]}$ or a Markov chain ${ }^{[56]}$. In [57], the $H_{\infty}$ filtering problem was investigated for a new class of discretetime networked nonlinear systems with both random delays (discrete delays and infinite distributed delay) and packet dropouts (Bernoulli distribution). Dong et al. ${ }^{[58]}$ studied the robust $H_{\infty}$ filtering problem for a class of uncertain nonlinear networked systems with both multiple stochastic time-varying communication delays and multiple packet dropouts. This work aimed to design a linear full-order filter such that the estimation error converged to zero exponentially in the mean square while the disturbance rejection attenuation was constrained to a given level by means of the $H_{\infty}$ performance index. The communication delays were regarded as a sequence of random variables which are mutually independent and obey Bernoulli distribution. The packet dropout phenomenon occurs with a certain probabilistic distribution in the interval $[0,1]$. The EKF is the most popular approach to recursive nonlinear estimation $^{[59-61]}$. In some simulations, it overweighs other sophisticated filters ${ }^{[61]}$. However, a large linearization error, the derivation of Jacobian matrices and limitation of Gaussian noise are three well-known drawbacks of the EKF. When the systems are strongly nonlinear and the noise is non-Gaussian, desired performance characteristics may not be obtained. In these cases, the particle filtering $(\mathrm{PF})$ methods proposed by Gorden in 1993 based on Monte Carlo simulation are available ${ }^{[62]}$. Recently, the PF has been extensively used in the fields of simultaneous localization and mapping (SLAM) for robots ${ }^{[63]}$ and visual tracking ${ }^{[64]}$. In [65], a continuous-discrete version of particle filter (CD-PF) for continuous-discrete nonlinear systems was proposed and some comparisons between CD-PF and CD-EKF were presented.

UKF is also a filtering method used for nonlinear systems. Kluge et al. ${ }^{[6]}$ analyzed the error behavior of the EKF, and models the arrival of the observation as a random process and a maximum dropout interval. $\mathrm{Li}$ and $\mathrm{Xia}^{[67]} \mathrm{ex}-$ tended the idea of [66] to the case of UKF, and presents an analysis of UKF for general nonlinear stochastic systems with intermittent observations. Under independent identical distribution packet dropouts, stability of the estimator may be analyzed by a modified discrete-time Riccati recursion effectively ${ }^{[66,67]}$. In contrast, this approach is not feasible for the Markovian packet dropouts model any more since the channel is described by several independent parameters, and the stability analysis faces more challenging. Next, we will give the detailed descriptions of some results about UKF.

Consider the discrete nonlinear system which is formulated as

$$
\begin{aligned}
& x_{k+1}=f\left(x_{k}, u_{k}\right)+w_{k} \\
& z_{k+1}=h\left(x_{k+1}\right)+v_{k+1}
\end{aligned}
$$

where $k \in \mathbf{N}$ is the discrete time, and $\mathbf{N}=\{0,1, \cdots\}$. $x_{k} \in \mathbf{R}^{n}$ is the state, $u_{k} \in \mathbf{R}^{m}$ is the control input, and $z_{k} \in \mathbf{R}^{q}$ is the measured output. The nonlinear functions $f$ and $h$ are continuously differentiable. $w_{k} \in \mathbf{R}^{w}$ and $v_{k} \in \mathbf{R}^{v}$ are the process and the measurement noise signal, respectively, which are assumed to be uncorrelated zeromean Gaussian white noise processes. $\mathrm{E}\left(w_{l} w_{k}^{\mathrm{T}}\right)=Q_{k} \delta_{k j}$ and $\mathrm{E}\left(v_{l} v_{k}^{\mathrm{T}}\right)=R_{k} \delta_{k j} . Q_{k}$ and $R_{k}$ are positive matrices. $x_{0}$ is uncorrelated with $w_{k}$ and $v_{k}$.

Case 1. The packet dropout is modeled as a Bernoulli process with parameter $\gamma \in[0,1]$.

Define a binary stochastic variable $\gamma_{k}$ as

$\gamma_{k}= \begin{cases}1, & \text { a measurement arrives after the } k \text {-th step } \\ 0, & \text { otherwise }\end{cases}$

and denote the variance of the output noise at time $k$ as $R_{k}$ if $\gamma_{k}=1$, and $\sigma^{2} I$ if $\gamma_{k}=0$ for some $\sigma^{2}$. Sinpoli et al. ${ }^{[42]}$ pointed out that the absence of an observation corresponds to the limiting case of $\sigma \rightarrow \infty$. The modified UKF is described as ${ }^{[67]}$ :

Step 1. Select sigma points. Assume that $x_{k}$ has mean $\bar{x}_{k}$ and covariance $\bar{P}_{k}$.

$$
\begin{aligned}
& \chi_{0, k}=\bar{x}_{k} \\
& \chi_{i, k}=\bar{x}_{k}+\left(a \sqrt{n} \bar{P}_{k}\right)_{i}, \quad i=1, \cdots, n \\
& \chi_{i, k}=\bar{x}_{k}-\left(a \sqrt{n} \bar{P}_{k}\right)_{i}, \quad i=n+1, \cdots, 2 n
\end{aligned}
$$

where $a$ is a proportion parameter and $\left(\sqrt{n} \bar{P}_{k}\right)_{i}$ is the vector of the $i$-th column of the matrix square root.

Step 2. Prediction.

$$
\begin{aligned}
& \chi_{i, k+1 \mid k}=f\left(\chi_{i, k}\right), \quad i=1, \cdots, 2 n \\
& \hat{x}_{k+1 \mid k}=\sum_{i=0}^{2 n} \varpi_{i} \chi_{i, k+1 \mid k} \\
& \hat{P}_{k+1 \mid k}=\sum_{i=0}^{2 n} \varpi_{i} \tilde{\chi}_{i, k+1 \mid k} \tilde{\chi}_{i, k+1 \mid k}^{\mathrm{T}}+Q_{k}
\end{aligned}
$$

where $\tilde{\chi}_{i, k+1 \mid k}=\chi_{i, k+1 \mid k}-\hat{x}_{k+1 \mid k}, \varpi_{0}=1-\frac{1}{a^{2}}, \varpi_{i}=\frac{1}{2 n a^{2}}$ $(i=1, \cdots, 2 n)$, and $\sum_{i=0}^{2 n} \varpi_{i}=1$.

Step 3. Update.

$$
\begin{aligned}
& z_{i, k+1 \mid k}=h\left(\chi_{i, k+1 \mid k}\right), i=0,1, \cdots, 2 n \\
& \hat{z}_{k+1 \mid k}=\sum_{i=0}^{2 n} \varpi_{i} \hat{z}_{i, k+1 \mid k} \\
& \hat{P}_{z z, k+1 \mid k}=\sum_{i=0}^{2 n} \tilde{z}_{i, k+1 \mid k} \tilde{z}_{i, k+1 \mid k}^{\mathrm{T}}+\gamma_{k+1} R_{k+1}+ \\
& \quad\left(1-\gamma_{k+1}\right) \sigma^{2} I \\
& \hat{P}_{x z, k+1 \mid k}=\sum_{i=0}^{2 n} \tilde{\chi}_{i, k+1 \mid k} \tilde{z}_{i, k+1 \mid k}^{\mathrm{T}} \\
& \hat{x}_{k+1}=\hat{x}_{k+1 \mid k}+\hat{P}_{x z, k+1 \mid k} \hat{P}_{z z, k+1 \mid k}^{-1}\left(z_{k+1 \mid k}-\hat{z}_{k+1 \mid k}\right) \\
& \hat{P}_{k+1}=\hat{P}_{k+1 \mid k}-\hat{P}_{x z, k+1 \mid k} \hat{P}_{z z, k+1 \mid k}^{-1} \hat{P}_{x z, k+1 \mid k}^{\mathrm{T}}
\end{aligned}
$$


where $\tilde{z}_{i, k+1 \mid k}=z_{i, k+1 \mid k}-\hat{z}_{i, k+1 \mid k}$. As $\sigma \rightarrow \infty$,

$$
\begin{aligned}
& \hat{x}_{k+1}=\hat{x}_{k+1 \mid k}+\gamma_{k+1} K_{k+1}\left(z_{k+1 \mid k}-\hat{z}_{k+1 \mid k}\right) \\
& \hat{P}_{k+1}=\hat{P}_{k+1 \mid k}-\gamma_{k+1} K_{k+1} \hat{P}_{x z, k+1 \mid k}^{\mathrm{T}} \\
& K_{k+1}=\hat{P}_{x z, k+1 \mid k}\left[\sum_{i=0}^{2 n} \tilde{z}_{i, k+1 \mid k} \tilde{z}_{i, k+1 \mid k}^{\mathrm{T}}+R_{k+1}\right]^{-1} .
\end{aligned}
$$

Step 4. Repeat Steps 1-3 for the next sample.

From Theorem 1 in [67], the boundedness of the estimation error for UKF is obtained as follows:

Theorem $1^{[67]}$. If the following conditions hold:

1) there exist real positive constants $\underline{f}, \underline{h}, \underline{\beta}, \underline{\alpha}, \bar{\alpha}, \underline{q}$, $\bar{q}, \underline{q}^{*}, \overline{\hat{q}}, \bar{r}, \underline{r}^{*}, \underline{p}, \bar{p}$, for every $k>0$, such that

$$
\begin{aligned}
& \underline{f}^{2} I \leq F_{k} F_{k}^{\mathrm{T}}, \quad \underline{h}^{2} \leq H_{k} H_{k}^{\mathrm{T}}, \quad \underline{\beta} I \leq \beta_{k} \\
& \underline{\alpha} \leq \alpha_{k} \leq \bar{\alpha} I, \quad \underline{q} I \leq Q_{k} \leq \bar{q} I, \quad \underline{q}^{*} I \leq Q_{k}^{*} \\
& R_{k} \leq \bar{r} I, \quad \underline{r}^{*} \leq R_{k}^{*}, \quad \underline{p} I \leq \hat{P}_{k} \leq \bar{p} I
\end{aligned}
$$

2) there exist real positive constants $\bar{f}, \bar{\phi}, \bar{h}, \bar{\beta}$ such that

$$
\begin{aligned}
& \left\|\gamma_{k} F_{k}\right\| \leq \bar{f}, \quad\left\|\phi_{k}\right\| \leq \bar{\phi} \\
& \left\|\gamma_{k} H_{k}\right\| \leq \bar{h}, \quad\left\|\beta_{k}\right\| \leq \bar{\beta}
\end{aligned}
$$

then the estimation error is exponentially bounded in mean square and bounded with probability 1 .

In addition, the error covariance matrices are also bounded for the modified UKF.

Theorem $2^{[67]}$. Assume that the linearized dynamic satisfies a modified uniform observability condition as in [66], and that the following conditions hold:

1) $\hat{P}_{1 \mid 0}>0$ and $H_{k}^{-1}$ exists and satisfies $\left\|H_{k}^{-1}\right\| \leq \underline{h}^{-1}$

2) there exists real positive constants $\underline{h}, \underline{\beta}, \bar{r}^{*}, \overline{\bar{\phi}}$, for every $k>0$, such that

$$
\begin{aligned}
& \underline{h}^{2} I \leq H_{k} H_{k}^{\mathrm{T}}, \quad \underline{\beta} I \leq \beta_{k}, \quad R_{k}^{*} \leq \bar{r}^{*} I \\
& Q_{k} \leq \bar{q} I, \quad\left\|\phi_{k}\right\| \leq \bar{\phi} .
\end{aligned}
$$

If $1>\gamma>\frac{\bar{\alpha}^{2} \bar{f}^{2}-1}{\bar{\alpha}^{2} f^{2} \phi^{2}}$, then there exists a upper boundedness $\bar{p}>0$ such that $\mathrm{E}\left(\hat{P}_{k+1}\right) \leq \bar{p} I$.

Case 2. The packet dropout is modeled as a Markov chain with a transition probability matrix as

$$
\Pi=\left(P\left\{\gamma_{k+1}=j \mid \gamma_{k}=i\right\}\right)_{i, j \in\{0,1\}}=\left[\begin{array}{cc}
1-q & q \\
p & 1-p
\end{array}\right]
$$

where $\gamma_{k}=1$ represents that the packet has been successfully transmitted to the filter, otherwise $\gamma_{k}=0$, and $0<p, q<1$.

$\mathrm{Li}$ and $\mathrm{Xia}^{[68]}$ considered the nonlinear system (16) with linear observation as

$$
z_{k}=H_{k} x_{k}+v_{k} .
$$

The modified UKF in [68] is similar to [67], in which the packet dropout is a Bernoulli process. Due to the linear observation equation, $\hat{P}_{z z, k+1}=H_{k+1} \hat{P}_{k+1 \mid k} H_{k+1}^{\mathrm{T}}+$ $\gamma_{k+1} R_{k+1}+\left(1-\gamma_{k+1}\right) \sigma^{2} I$ and as $\sigma \rightarrow \infty, K_{k+1}=$ $\hat{P}_{x a, k+1}\left[H_{k+1} \hat{P}_{k+1 \mid k} H_{k+1}^{\mathrm{T}}+R_{k+1}\right]$. The boundedness of the estimation error and the boundedness of the estimation error covariance matrices were obtained in [68].

Under some conditions (see Assumption 1 in [68]), the mean of the estimation error $\mathrm{E}\left(\left\|\tilde{x}_{k}\right\|^{2}\right)$ is bounded by $\eta \mathrm{E}\left(\left\|\tilde{x}_{0}\right\|^{2}\right)(1-\lambda)^{n}+\nu$, where $\eta, \nu>0$.

Under the condition that $\left(F_{k}, H_{k}\right)$ is uniform observable, the stability in stopping times and the sampling times is given respectively as

1) Stability in stopping times: If the recovery rate $q$ satisfies that $\bar{\alpha}^{2} \bar{f}^{2}(1-q)<1$, then $\sup _{k \in \mathbf{N}} \mathrm{E}\left[\left\|\hat{P}_{\tau_{k} \mid \tau_{k-1}}\right\|\right]<\infty$.

2) Stability in sampling times: If the conditions $\bar{\alpha}^{2} \bar{f}^{2}+$ $\bar{\sigma} \bar{\alpha}^{2} \bar{f}^{2}+2 \bar{\sigma} \bar{h}^{2} \bar{g}^{2}<1$ and $P_{1 \mid 0}>0$ are satisfied, then $\sup _{k \in \mathbf{N}} \mathrm{E}\left[\left\|\hat{P}_{k \mid k-1}\right\|\right]<\infty$.

The details can be referred to Theorems 1 and 2 in [68].

In fact, there exist fruitful results about filtering for the general system. When network is adopted as the communication medium, the previous filtering methods are improved to deal with the network-induced problems, such as delay, packet loss, etc. In the future, in the filter design process, more practical factors, such as bandwidth, network load and sensor saturation, should be taken into consideration.

\subsection{Data fusion}

Data fusion is a broad research area, which includes detection fusion, image fusion, data association, estimation fusion, etc. In this section, data fusion mainly refers to estimation fusion. In addition to the filtering, estimation fusion is another important approach to estimation. In general, filtering often deals with the data from single sensor. In contrarily, estimation fusion is the estimation which involves multi-sensor data. Multi-sensor data fusion has been one of the key technologies of networked control systems, which have been served as an interesting benchmark in the past decades. The aim of multi-sensor data fusion is to increase the accuracy of the estimation. It has been applied to both military field and nonmilitary field. Military applications include automated target recognition, guidance for autonomous vehicles, remote sensing, battlefield surveillance, and automated threat-recognition systems, such as identification-friend-foe-neutral systems. Nonmilitary applications include monitoring of manufacturing processes, condition-based maintenance of complex machinery, robotics, and medical applications.

Multi-sensor data fusions can be divided into three categories:

1) Centralized data fusion. It is also called measurement fusion. All the measurements from different sensors are transmitted to the fusion center and dealt within the fusion center. Due to the full involvement of all the measurements, this method is optimal. The drawback is stringent requirements for bandwidth of the channels, enormous energy consumption and processor with high performance in the fusion center.

2) Distributed data fusion. It is also called state fusion. After locally preprocessing the measurement of each sensor, the results are sent to the fusion center. This method not only decreases the demand for the channel, but 
also has a strong fault tolerance ability.

3) Hybrid data fusion. This method is the combination of the centralized data fusion and the distributed data fusion. However, the communication cost and the computation cost are high.

In the following, we will mainly present the recent results of the centralized data fusion and the distributed data fusion, respectively. Sequently, considering the unreliability of the network, data fusion in NCSs is also introduced in the end of this subsection.

\subsubsection{Centralized data fusion}

The early results about centralized data fusion were relatively simple. Singer and Kanyuck ${ }^{[69]}$ firstly studied the problem of the multi-sensor state estimation. However, it was assumed that the estimation error of each sensor is mutually independent. Willner et al. ${ }^{[70]}$ proposed a centralized multi-sensor KF algorithm. Afterwards, in general, more scholars studied the systems in which the noise of each sensor is correlated. The centralized information integration architecture proposed in [71] is based on the output augmented fusion method. The usual approach in the classical EKF implementation is based on the assumption of constant diagonal matrices for both process and measurement covariances. A new adaptive modified EKF (AMEKF) algorithm has been developed to prevent the filter divergence.

The centralized data fusion has been widely applied. Munz et al. ${ }^{[72]}$ constructed a multi-sensor fusion system which was implemented to be independent of both sensor hardware properties and application requirements in the driver assistance system. This supports changes in sensor combination or application requirements. Moreover, the environmental model can be used at the same time. A probabilistic approach for this generic centralized fusion system was presented and discussed. In [73], a centralized fusion scheme was adopted for obstacle and road tracking. A KF based curvilinear model predicted drivers' behavior and the system was able to assess the level of threat for all moving obstacles and decide the warning policy. The proposed approach has been tested with real data to show the performance of the tracking system.

\subsubsection{Distributed data fusion}

As we know, the centralized data fusion usually has the optimal performance for making most of all the information. Relatively, the distributed algorithm sacrifices the optimal performance to increase the flexibility and decrease the communication cost. If the distributed data fusion is said to be optimal, compared to the centralized data fusion, it often has the approximate optimal performance under some assumptions or conditions. For early results about distributed data fusion, please refer to [74-77]. BarShalom ${ }^{[78]}$ proposed that there exits the same process noise in the dynamic of different sensors. Thus, the estimation of each sensor is mutually dependent. Based on this, BarShalom ${ }^{[78]}$ presented a distributed state fusion algorithm and discussed the influence of process noise on the variance of the estimation error. Grime et al. ${ }^{[79]}$ considered that there is no central fusion center and no single sensor node which can obtain global knowledge of network and designed a decentralized data fusion scheme with the information of each's nearest communication neighbor. For the past decade, distributed data fusion has attracted considerable attention. In [80], an architectural framework of distributed data fusion was developed and it consisted of a data fusion application programming interface (API) and a distributed algorithm for energy-aware role assignment. Mahmoud and $\mathrm{Xia}^{[81]}$ studied the distributed filtering and fusion in the wireless sensor network. In [82], support vector machines were used to compress the probabilistic information available in the form of independent identical distribution samples and to solve the Bayesian data fusion problem.

Distributed data fusion has also been applied to many fields. Regazzoni and Tesei ${ }^{[83]}$ described an approach to estimating the number of pedestrians present in a real-life complex scene by processing a sequence of images. The proposed estimation approach exploits temporal information by means of a distributed KF network. In [84], distributed estimation algorithms based on the information graph were presented for arbitrary fusion architectures and related to linear and nonlinear distributed estimation results and the proposed approaches were applied to tracking.

\subsubsection{Data fusion in NCSs}

Due to the existence of the network-induced delay, the packet dropout and other characteristics in the network, the traditional data fusion techniques are not proper and some new data fusion approaches for NCSs were studied. In the following, we will introduce some advances for the unreliable networks.

Besada-Protas et al. ${ }^{[85]}$ proposed a set of new centralized algorithms for estimating the state of linear dynamic multiple-input multiple-output control systems with asynchronous, non-systematically delayed and corrupted measurements provided by a set of sensors. A streamlined memory and computational efficient reorganization of the basic operations of the Kalman and information filters (KF \& IF) were designed. In [86], the optimal distributed fusion update algorithm with multiple local asynchronous (1-step-lag) out-of-sequence-measurement (OOSM) update was firstly deduced, which was proved, under some regularity conditions, to be equivalent to the corresponding optimal centralized update algorithm with all-sensor 1-step-lag OOSMs. Reference [87] was concerned with the distributed $\mathrm{KF}$ problem for a class of networked multi-sensor fusion systems (NMFSs) with missing sensor measurements, random transmission delays and packet dropouts. An optimal distributed fusion KF (DFKF) was designed based on the optimal fusion criterion weighted by matrices. In [88], a new networked multi-sensor data fusion method which was based on federated filter is proposed.

In [89], a networked data-fusion approach with packet losses and variable delays was considered and a federated filter was employed to fuse the data transmitted over the network, which plays an important role in the data-processing center. The federated filters are of peak covariance stability if the delayed data is processed by the proposed algo- 
rithm. Zhu et al. ${ }^{[90]}$ proposed a centralized architecture and a distributed architecture with buffer for the problem of networked data. A modified KF was adopted to estimate the state with measurement delay and loss. In [91], a centralised data fusion approach was presented for the system, in which the measurements of the local sensors are timestamped, and then transmitted through the network to the fusion centre. A buffer was designed to deal with the measurement delay or loss. A probabilistic metric to evaluate the performance of the system was employed.

Multi-rate framework has caused the interests of many scholars because of the decrease of the communication cost. Fig. 4 shows an example of multiple sampling rates. Zhang et al. ${ }^{[92]}$ presented a distributed fusion estimation method for estimating states of a dynamical process observed by wireless sensor networks (WSNs) with random packet losses. It was assumed that the dynamical process is not changing too rapidly, and a multi-rate scheme by which the sensors estimate states at a faster time scale and exchange information with neighbors at a slower time scale is proposed to reduce communication costs. Liang et al. ${ }^{[93]}$ presented a multi-rate filtering problem for a class of networked multi-sensor fusion systems with packet dropouts. Different from the single-rate estimator design with packet dropouts which are treated as stochastic parameters, an unknown input observer was proposed, where packet dropouts are represented as zero-mean white input noises of the linear time-variant estimation error system. Yan et al. ${ }^{[94]}$ considered the state estimation for a kind of nonlinear multirate multisensor asynchronous sampling dynamic system. $N$ sensors observed a single target independently at multiple sampling rates, and the dynamic system was formulated at the highest sampling rate. The multiscale system theory and the modified sigma point KF were used to design the fusion method. Measurements randomly missing with Bernoulli distribution could also be allowed in [94].

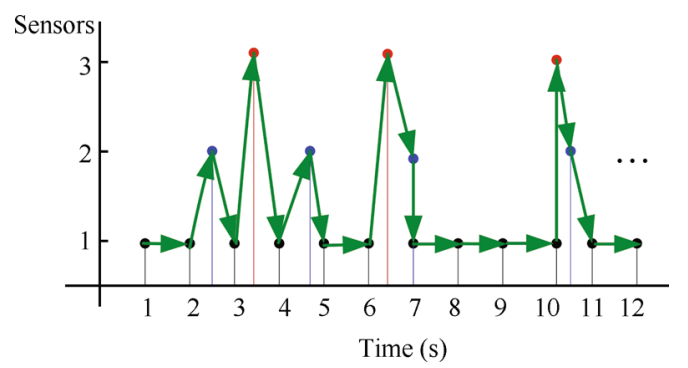

Fig. 4 An example of multiple sampling rate

There are also some significant results which are concerned with the communication constraints and energy consumption. In [95], the realistic sensing/reporting channels and the correlated log-normal shadow-fading in all wireless links of the cooperative network were considered. It was shown that shadowing correlation on the reporting channel can yield performance degradations similar to the shadowing correlation on the sensing channel. In [96], the problem of optimal state estimation was concerned for linear systems when the noises of different sensors were cross-correlated and also coupled with the system noise of the previous step. Bian et al. ${ }^{[97]}$ considered the medium access constraint. By introducing a stochastic process, a new model was developed that can truly reflect this contradiction (the number of transmitted sensors and delay steps). The upper bounds of the expected estimation error covariance and estimation error covariance with one-step delay are $\mathrm{E}\left(\left[P_{t \mid t-1}\right]\right) \leq \bar{N}_{P r}$ and $P_{t \mid t-1}<\bar{M}$, respectively, where $\bar{N}_{P r}=A\left(C_{1}^{\mathrm{T}} R_{1}^{-1} C_{1}\right)^{-1} A^{\mathrm{T}}+Q$ and $\bar{M}=A\left(C_{0}^{\mathrm{T}} R_{0}^{-1} C_{0}\right)^{-1} A^{\mathrm{T}}+Q$. In [98], energy efficient state estimation was considered. The packet loss probability depended upon time-varying channel gains, packet lengths and transmitted power of sensors. The sufficient conditions, imposing constraint on the packet loss probability of each channel were established by taking into account each observable subsystem structure to guarantee that the expectation of the trace of estimation error covariance matrices is exponentially bounded, and the upper bound is $\mathrm{E}\left(\operatorname{tr}\left(P_{t \mid t}\right)\right) \leq \frac{1}{S} \sigma_{\max }^{2}\left(B_{o}^{-1}\right) \sum_{i=1}^{S}\left[\rho_{i}^{t} \operatorname{tr}\left(P_{0 \mid 0}^{i}\right)+\right.$ $\left.\frac{\eta_{i}}{\rho_{i}}\right], \quad$ where $B_{o}=\operatorname{col}\left(B_{o}^{1}, B_{o}^{1}, \cdots, B_{o}^{S}\right), \quad \eta_{i}=$ $\operatorname{tr}\left(A_{o}^{i}\left(C_{o}^{i}\right)^{-1} R^{i}\left(C_{o}^{i}\right)^{\prime-1}\left(A_{o}^{i}\right)^{\prime}\right) \operatorname{Pro}\left(\gamma_{t}^{i}=1\right)+\operatorname{tr}\left(B_{o}^{i} Q\left(B_{o}^{i}\right)^{\prime}\right)$, $\rho_{i}=\left(1-\operatorname{Pro}\left(\gamma_{t}^{i}=1\right)\right) \alpha_{i}, \alpha_{i}=\sigma_{\max }^{2}\left(A_{o}^{i}\right)$ for $i=1,2, \cdots, S$, $B_{o}^{i}$ and $A_{o}^{i}$ are the matrices related to the sensors, $Q$ and $R_{i}$ are the covariance matrices, $\sigma_{\max }$ is the largest singular value and $\gamma_{t}$ is the transmission outcome of the sensor.

Up to now, filtering for NCSs with single channels has been researched widely. However, data fusion is still a novel research branch. The existing approaches mainly involve the linear systems, in which some priori statistical characteristics of measurement are given and mostly the disturbances in different sensors are assumed to be uncorrelated. In the future, the optimal fusion method for nonlinear system needs to be designed in the presence of parameter uncertainty. Moreover, the correlation between the disturbances should also be taken into account.

\section{Fault diagnosis of NCSs}

In this section, we mainly summarize the progress on fault diagnosis of NCSs. Essentially, fault diagnosis is the process in which the available information in the diagnosis system is processed to generate the running state and the fault condition of the system. Different from the traditional fault diagnosis, the fault diagnosis of NCSs has the following characters:

1) Because the data transmitting in the network channel is digital quantity, the model of the system is of the discrete form.

2) The transmission delay of the system is non-ignorable.

3) Since there are different node drive forms and different data transmission methods in the network, the corresponding models of the system have different forms.

4) The model of the controlled object is always nonlinear.

5) The packet dropout in NCSs brings a negative effect to the fault diagnosis. 
Some recent progress about fault diagnosis will be shown in the following. For the fault diagnosis system, two negative factors need to be considered: the uncertainty or the disturbance and the known input induced by the delay. In [99], the influence of network-induced delay on conventional observer based fault detection systems was first investigated. However, this method cannot meet the requirements for effective residual information. To solve this problem, Zhang et al. ${ }^{[100]}$ presented that if the unknown network-induced delay is less than the sampling period, an NCS with disturbance and fault can be regarded as the NCS without delay. Moreover, the effect of the network-induced delay can be seen as an unknown input. The fault diagnosis system was robust to this input. In [101], a relationship between the parity relation and the $H_{2}$ optimal residual generators was established, and the optimal parity vector $H_{2}$ converged to the $H_{2}$-optimal post-filter with $s \rightarrow \infty$. The effect of the unknown input was decreased. When the delay is larger than the sampling period, the traditional method is not effective. For this problem, in [102], the problem of robust fault estimation for a class of uncertain NCSs with random communication network-induced delays was investigated. Based on the Lyapunov-Razumikhin method, Huang and Nguang ${ }^{[102]}$ gave a delay-dependent fault estimator in terms of the solvability of bilinear matrix inequalities (BMIs) and proposed an iterative algorithm to change this non-convex BMI problem into quasi-convex optimization problems. In this work, delay was not required to be less than the sampling period, but bounded.

In order to rationally utilize network resources and reduce the network congestion and delay, Xie et al. ${ }^{[103]}$ constructed a simple information-scheduling scheme to describe the scheduling approach of system signals in NCSs. For the sake of the optimization, the scheme made the NCSs share the network resource periodically. Considering the short delay in the network, Ye et al. ${ }^{[99,101,104-106]}$ utilized the state estimation and the observer theory to reduce the effect of the delay. A lowpass filter and the Cayley-Hamilton based approach were proposed in these works. Huo and Fang ${ }^{[107-110]}$ studied the fault diagnosis systems with random delays and communication constraints. In addition, for the NCSs with large delay, Fang et al. ${ }^{[111-113]}$ used quasi Takagi-Sugeno fuzzy model to design the fault diagnosis approach. These works not only considered the influence of the network-induced delay, but also investigated the effect of the packet loss. Furthermore, Mao et al. ${ }^{[114]}$ studied the problem of protocol and fault detection for nonlinear NCSs.

In addition, Liu et al. ${ }^{[15]}$ researched the fault detection of linear systems over networks with bounded packet loss. The packet loss process is assumed to be arbitrary or Markovian. For a bounded packet loss rate, a switched system was constructed to be the monitored system by resampling it at each time instant when the measurements arrive at the fault detection node. This work designed a filter for the switched system, which updates only at the time instant when new measurements arrived at the fault detection node. Similarly, the input data packets' lost were con- sidered as external disturbances. In [116], fault detection for Takagi-Sugeno fuzzy discrete systems in finite-frequency domain was studied. Faults were considered in a middle frequency domain, while disturbances were considered in another certain finite-frequency domain interval. The filter design was formulated as a two-objective optimization algorithm. Liu and $\mathrm{Xia}^{[117]}$ presented a fault detection and compensation scheme based on likelihood ratios (LRs) for networked predictive control systems with random networkinduced time delays and clock asynchronism. The compensator eliminated the effect of the random delay. In order to reduce the influence of the asynchronism, the observer updated based on the time schedule of a remote node clock. Two frameworks were designed to update the LRs of fault. The convergence analysis of a generalized LR test with intermittent observations was given. Xia and $\mathrm{Liu}^{[118]}$ considered the detection of abrupt changes for MIMO linear systems based on frequency domain data. The measured inputs and outputs were maped from the time domain to the frequency domain by using the real discrete-time Fourier transform. The latent state change was modeled as an initial state disturbance to be estimated on the basis of frequency domain samples. In this work, the occurrence time was estimated by maximizing a likelihood ratio function. In [119], the detection of abrupt change was used in electrocardiogram. An linear time varying (LTV) model with Gaussian white noise was used to describe the real electrocardiogram signal. Based on the estimated system parameters and tuned covariances of noise, the generalized likelihood ratio (GLR) tests for electrocardiogram signal were developed for abrupt change detection.

To sum up, there are relatively fewer results for fault diagnosis of nonlinear NCSs. How to extend the achievements for linear systems to nonlinear systems, especially considering the network-induced delay and the uncertainty simultaneously, is still a difficulty at the current stage. In addition, the affects of network protocol for the fault diagnosis should also be emphasized in the future.

\section{$5 \quad$ Networked predictive control}

Due to the existence of the network, the traditional control strategies are not suitable for the novel networked control systems. Numerous control approaches for NCSs have attracted the interests of the researchers. These approaches aim to deal with the time delay and the packet dropout. Chou and Tipsuwan ${ }^{[120]}$ divided the recent networked control techniques into five classes (augmented deterministic discrete-time model method, queuing method, optimal stochastic control method, perturbation method and sampling time scheduling method). In the following, we will present the main results about networked predictive control (NPC). First, MPC for NCSs is summarized and the framework to deal with delay and dropout using MPC is constructed. Then, the predictor, based on a sequence of past measurements, is called data package based predictor here. The corresponding predictive method is 
called data package based predictive control and the results of this method are shown. Thirdly, when the system is extremely complex, the model of the system cannot be obtained. What can be used are only the input and output. Consequently, the data-driven predictive control is proposed. Up to now, there are few achievements about this aspect. The main idea is presented in this survey. With the increase of the complexity of the task, distributed cooperative control for NCSs is studied and a considerable number of distributed control techniques were studied in the past years. Due to the space limit, in the end of this section, we only discuss some important results of the distributed predictive control for NCSs.

\subsection{Model predictive control for NCSs}

MPC, which is also called receding horizon control ( $\mathrm{RHC})$, is a form of control in which the current control action is obtained by solving on-line, at each sampling instant, a finite horizon open-loop optimal control problem, using the current state of the plant as the initial state. The optimization yields an optimal control sequence and the first control in this sequence is applied to the plant ${ }^{[121]}$. For the past decades, it has gained notable attention in control of dynamic systems and has played an important role in control practice. One of the significant advantages for MPC is the capability of handling the constraints. Due to the existence of the constraints, the traditional determinate controller may result in constraint violations. However, in the MPC framework, the constraints are regarded as one part of the optimization problem and can be handled properly. Another merit of MPC is the optimality of the solution in some meaning. The objective function of MPC often represents the cost of the state, the control input and other items with different senses. In most cases, the analytical solution cannot be provided and only the local optimal numerical solution can be gained.

Consider a nonlinear discrete-time system

$$
x(k+1)=f(x(k), u(k)) .
$$

MPC can be formulated as

$$
J^{*}\left(k, x^{*}, u^{*}\right)=\min _{u(\cdot \mid k)} J(k, x, u)
$$

with

$$
\begin{gathered}
J(k, x, u)=\sum_{l=0}^{N-1}(L(x(k+l \mid k), u(k+l \mid k)))+ \\
F(x(k+N \mid k))
\end{gathered}
$$

subject to

$$
x(k \mid k)=x(k)
$$

for all $l=0, \cdots, N-1$

$$
\begin{aligned}
& x(k+l+1 \mid k)=f(x(k+l \mid k), u(x+l \mid k)) \\
& x(k+l \mid k) \in \mathbb{X} \\
& u(k+l \mid k) \in \mathbb{U} \\
& x(k+N \mid k) \in \Omega
\end{aligned}
$$

where $X$ is admissible state set, $U$ is the admissible control set and $\Omega$ is the terminal set.

In general, the cost function has a quadric form, i.e., $L(x(k+l \mid k), u(k+l \mid k))=\|x(k+1 \mid k)\|_{Q}^{2}+\|u(k+l \mid k)\|_{R}^{2}$ and $F(x(k+N \mid k))=\|x(k+N \mid k)\|_{P}^{2}$. In order to ensure the stability and the feasibility, $Q, R$ and $P$ need to satisfy some conditions. More details about them can be found in $[121,122]$.

For NCSs, MPC has been used as a significant tool to deal with the negative effect which the delay and the dropout induce. In the following, we will summarize MPC for NCSs without constraints and with constraints, respectively.

When only considering the delay and the dropout, many works about MPC can be found. Irwin et al. ${ }^{[123]}$ presented a co-design approach to wireless network control systems and Lyapunov stability was derived using LMIs for the multiobserver linear quadratic (LQ) control. Liu et al. ${ }^{[124]}$ proposed a modified MPC, which uses the future control sequence to compensate for the forward communication time delay, to deal with networked control systems with random communication time delay. For both fixed and random communication time delays, if there exists $P$ such that $\Lambda^{\mathrm{T}}(\tau) P \Lambda(\tau)<P$ where $\Lambda(\tau)$ is the closed-loop system matrix, the closed-loop is stable. In [125], the predictive control and the integral control were used to compensate for the network link effects and eliminate the tracking error. Zhao et al. ${ }^{[126,127]}$ designed an improved predictive controller using delayed sensing data and proposed a compensation scheme to overcome the negative effects of the network-induced delays and data packet dropouts in both forward and backward channels.

In addition, some achievements for NCSs with constraints can be found. In [128], the input and the output were constrained by the nonlinear formulations. MPC based on the mixed-integer programming was used to derive the controller. It turned out that the controller design was feasible due to the special nature of the data transmission strategy, only a small number of logic values was involved. In order to minimize bandwidth utilization, Goodwin et al. ${ }^{[129]}$ imposed a communication constraint which restricted all transmitted data to belong to a finite set and only permited one plant to be addressed at a time. The moving horizon approach was used to deal with both control and measurement quantization issues. Varutti et al. ${ }^{[130]}$ proposed an event-based model predictive control approach to nonlinear continuous time systems under state and input constraints. The method can counteract bounded delays, information losses, as well as deal with event triggering due to sensors and actuators. Quevedo and Nešićc ${ }^{[31]}$ 
studied an MPC scheme in which packets containing optimizing sequences of control inputs were sent over an unreliable communication network with packet loss. MPC for nonlinear systems subject to packet losses was proposed in [132]. The proposed controller allowed for an explicit characterization of the stability region and it turned out that this region was an invariant set for the closed-loop system with packet losses. In [133], a robust MPC with a network delay compensation strategy was proposed for uncertain discrete-time constrained nonlinear systems with time-varying transmission delays and packet dropouts. Xia et al. ${ }^{[134]}$ considered robust constrained MPC of systems with polytopic uncertainties. Sufficient conditions for the existence of parameter-dependent Lyapunov functions were proposed in terms of LMIs, which would reduce the conservativeness resulted from using a single Lyapunov function. The combination of event-triggering control and MPC was also used for $\mathrm{NCSs}^{[135-138]}$. A novel event-triggered strategies was proposed in [137] for the control of uncertain nonlinear systems with additive disturbances under robust MPC. The main function of the event-triggered scheme was to trigger the solution of the optimal control problem of the robust MPC.

Other results can be found in [139-152].

\subsection{Data package based predictive control for NCSs}

The fundamental idea and the basic framework of the data package based predictive control will be shown firstly. The discrete dynamic system is considered as

$$
\begin{aligned}
& x(k+1)=f(x(k), u(k), w(k)) \\
& y(k)=g(x(k), u(k), v(k))
\end{aligned}
$$

where $x(k)$ is the system state, $u(k)$ is the system input, $y(k)$ is the system output, $f(\cdot)$ and $g(\cdot)$ are the system equations, which can be linear or nonlinear, $w(k)$ is the unknown process disturbance, and $v(k)$ is the unknown measurement noise.

Package based predictive control has been proved to be an effective method for networked control systems with network-induced time delay and data dropouts ${ }^{[153,154]}$. The framework of package based predictive control system is shown in Fig. 5. It's worth noting that, in order to satisfy that the measurements are processed in sequence, a buffer is set at the controller node. The estimated states and predictive states can be obtained by using KKF:

$$
\begin{aligned}
& \hat{x}(k \mid k)=K F(S, \hat{u}(k-1 \mid k-1), y(k)) \\
& \hat{x}(k+i \mid k)=K F(S, \hat{u}(k \mid k), y(k)), i=1,2, \cdots, N_{1} \\
& \hat{u}(k+i \mid k)=K(k+i) \hat{x}(k+i \mid k), i=1,2, \cdots, N_{1}
\end{aligned}
$$

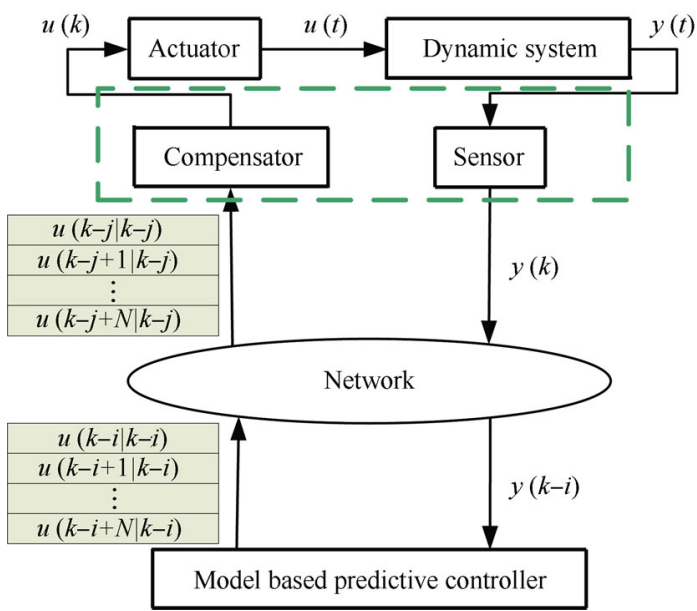

Fig. 5 Date packing based predictive control for NCSs

where $N_{1}$ is the finite $\mathrm{KF}$ gain ${ }^{[154]}$. The processed states are then sent to the controller node.

In general, there inevitably exist the unknown network transmission delays in both the forward (from controller to actuator) channel (CAC) and feedback (from sensor to controller) channel (SCC). A networked predictive control scheme mainly consists of the control prediction generator and the network delay compensator. The former is designed here to generate a set of future control predictions, while the later is used to compensate for the unknown random network delays. If the network can transmit a set of data at the same time, it is assumed that a predictive control sequence at time $k$ is packed and then sent to the plant side through a network. In the compensator node, the latest control value from these control sequences will be chosen on the plant side.

For example, when the time delays in SCC and in CAC are respectively 0 and $k_{i}$, given that the following control predictive sequences are received on the plant side:

$$
\begin{aligned}
& {\left[u_{t-k_{1} \mid t-k_{1}}^{\mathrm{T}}, u_{t-k_{1}+1 \mid t-k_{1}}^{\mathrm{T}}, \cdots, u_{t \mid t-k_{1}}^{\mathrm{T}}, \cdots, u_{t+N-k_{1} \mid t-k_{1}}^{\mathrm{T}}\right]^{\mathrm{T}}} \\
& {\left[u_{t-k_{2} \mid t-k_{2}}^{\mathrm{T}}, u_{t-k_{2}+1 \mid t-k_{2}}^{\mathrm{T}}, \cdots, u_{t \mid t-k_{2}}^{\mathrm{T}}, \cdots, u_{t+N-k_{2} \mid t-k_{2}}^{\mathrm{T}}\right]^{\mathrm{T}}} \\
& \cdots \\
& {\left[u_{t-k_{t} \mid t-k_{t}}^{\mathrm{T}}, u_{t-k_{t}+1 \mid t-k_{t}}^{\mathrm{T}}, \cdots, u_{t \mid t-k_{t}}^{\mathrm{T}}, \cdots, u_{t+N-k_{t} \mid t-k_{t}}^{\mathrm{T}}\right]^{\mathrm{T}}}
\end{aligned}
$$

where the control sequences $u_{t \mid t-k_{i}}, i=1,2, \cdots, t$, are available to be chosen as the control input of the plant at time $t$. The output of the network delay compensator, i.e., the input of the actuator will be

$$
u_{t}=u_{t \mid t-\min \left\{k_{1}, k_{2}, \cdots, k_{t}\right\}} \cdot
$$

In fact, by using the networked predictive control scheme presented in this section, the control performance of the closed-loop system with network delay is very similar to that of the closed-loop system without network delay.

Next, we will show some results about package based predictive control ${ }^{[155-171]}$. Xia et al. ${ }^{[155]}$ studied the stochastic stability analysis of networked control systems with random 
network delay. A scheme, including control prediction generator and network-delay compensator, was proposed. The control prediction generator, which makes the closed-loop system achieve the desired control performance, was used to generate a set of future control predictions and the networkdelay compensator removed the effects of the network transmission delay and data dropout. In [161], random network delay in the feedback channel was considered and stability criteria of closed-loop networked predictive control systems was given. Liu et al. ${ }^{[167]}$ considered both constant and random network delays in the forward and feedback channels. A networked predictive control scheme was proposed to overcome the effects of network delay and data dropout. The necessary and sufficient conditions for the stability of closed-loop NCS with constant time delay were given. Xia et al. ${ }^{[170]}$ proposed the switched system's method, which solves the stochastic problem in a deterministic way.

\subsection{Data-driven predictive control for NCSs}

According to traditional control design frameworks, control and monitoring need support from dynamic models. In general, the state or the output measurements from the plant are the most significant elements to build the system model. When finishing the design of a controller or a monitor, the model will no longer exist. However, while using models in this framework, the modeling error and the complexity in building the model cannot be neglected. Specifically, with Internet of things (IOT), big data needs to be processed urgently. And inevitably, the complexity of the traditional control frameworks will continue to increase. To address these issues, the data-driven scheme was developed, which has been applied in the industrial process control field as well as in complex systems. Further more, if the digital data flows through the network, which connects the controller and the actuator, then the data-driven method is proper for NCSs, especially when the system model is hard to build.

In the following, the notions of data-driven predictive networked control system are introduced. The typical structure of data-driven predictive networked control system is shown in Fig. 6. In such a control system, the predictive control signals are generated by the subspace projection method. And data-driven predictive networked control systems and data package based predictive control systems differ only in the controller. In data-driven networked control systems, when the past input and past output from the sensors are transmitted to the controller, the data-driven predictive control algorithm will be used to generate a sequence of predictive control inputs. And then those control sequences will be transmitted to a buffer at the actuator site over the network. At last, according to the predictive networked control scheme described in (47) and (48), the compensator will select the proper control input. From the description above, the data driven networked control scheme can be obtained directly without any model. And, it is obvious that this framework is different from the data package based predictive method in [155].

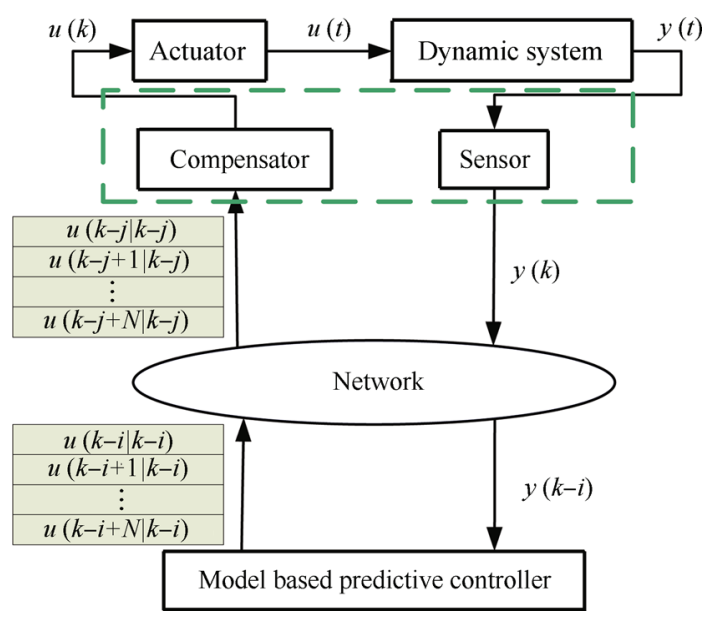

Fig. 6 Data driven predictive control for NCSs

Up to now, there are some improvements of data-driven predictive control ${ }^{[172-175]}$. Laurí et al. ${ }^{[173]}$ proposed modelbased predictive control methodology in the space of the latent variables for continuous processes. Xia et al. ${ }^{[175]}$ proposed data-driven predictive control for networked control systems, which was designed by applying the subspace matrices technique and obtained directly from the input/output data transferred from networks. Implementing identification and control in the latent variable space is easier than in the case of correlation in the data set. It can act as a prefilter to reduce the effect of noisy data, and can finally reduce computational complexity.

\subsection{Networked distributed predictive con- trol}

In this subsection, we mainly emphasize the distributed model predictive control. The considerable increase of the computational power of the hardware and the rapid development of the communication technology bring novel opportunity to the decentralized or distributed MPC $(\mathrm{DMPC})^{[176-185]}$. Maestre and Negenborn ${ }^{[186]}$ mainly introduced a comprehensive overview distributed MPC and contributed to answering the question what kind of distributed model-based control technique could be appropriate for control of large-scale intermodal networks, or parts thereof. Negenborn and Maestre ${ }^{[187]}$ presented the main features of distributed MPC in three aspects (process features, control architecture features and theoretical features) and provided the future development of DMPC. Eventbased DMPC was explored in $[188,189]$ and it was applied to a multi-agent system. In general, for DMPC, there are three update approaches: synchronous DMPC, sequential DMPC and iterative DMPC. In synchronous DMPC scheme, all the agents solve the optimization problem at the same time ${ }^{[184,185,190-192]}$. In the sequential DMPC framework, all the agents solve the optimization problem in the specified order ${ }^{[180-182]}$. The advantage of the sequen- 
tial scheme is that less communication and computation is needed, as compared with the synchronous one. In the iterative DMPC, each agent solves its optimization problems and communicates the solution with its neighbors iteratively until the local convergence of the solution.

The stability and feasibility of the DMPC are the significantly challenging issues. When the goal region of the system is a set of certain setpoints, many theoretical and applied results were proposed ${ }^{[182-184,190-192]}$. Richards and How ${ }^{[182]}$ proposed a formulation of robust DMPC for the systems with coupled constraints and unknown but bounded additive disturbances. However, if the set to converge is a dynamic region such as consensus or synchronization and tracking, there are some issues in DMPC framework. In [183], a general DMPC framework for cooperative control was considered and an algorithm to test the satisfaction of the terminal region was formulated. However, all the subsystems share a public terminal region and the terminal region is possibly conservative. Wang and Ding ${ }^{[184]}$ proposed a positively invariant time-varying terminal state region with the corresponding auxiliary controller to achieve the multi-vehicle formation control. Dunbar and Murray ${ }^{[190,191]}$ divided the centralized MPC (CMPC) scheme into DMPC one. Then, lower bounds on the update rate and upper bounds on the mismatch between actual and assumed trajectories were established to achieve multi-agent formation stabilization.

Considering the communication delay and the noise, there are only a few works ${ }^{[193-195]}$. Li and Shi ${ }^{[194]}$ studied the robust DMPC problem for a group of nonlinear agents (subsystems) subject to control input constraints and external disturbances. A robustness constraint was designed to deal with external disturbances. The feasibility of the robust DMPC scheme and the robust stability of the overall agent system were analyzed, respectively. $\mathrm{Li}$ and $\mathrm{Shi}^{[195]}$ proposed the robust DMPC for large-scale continuous-time nonlinear systems subject to communication delays and external disturbances. They proved that 1) the bounds of external disturbances, the sampling period and the bound of communication delays all affect the feasibility; 2) the stability is related to the bounds of external disturbances, the sampling period, the bound of communication delays and the minimum eigenvalues of the cooperation matrices; 3 ) the closed-loop system is stabilized into a robust invariant set under the proposed conditions.

In addition to distributed networked predictive control, other distributed networked control approaches were also proposed for the cooperation of multiple NCSs. Take the application of multiple rigid spacecrafts as an example. The compound control methodology for flight vehicles was proposed in [196]. Zhou et al. ${ }^{[197-200]}$ mainly studied the synchronization and tracking control of multiple rigid spacecraft with uncertainty and disturbance. Finite-time terminal sliding mode technique was adopted in these works. Due to the limitation of space, other distributed approaches will not be presented here.

In summary, networked predictive control is an important approach to NCSs. Currently, most existing researches depend on the system model, especially the linear one. When using the nonlinear predictive control, the stability of the NCSs is still a challenge. Data-based control is a novel research direction, which can decrease the dependence on the accurate model. The research for data-driven predictive control is at its initial stage and there are many theoretical problems to be handled. In addition, distributed predictive schemes have been adopted in NCSs, such as the multiagent systems. However, when the delay and the packet loss are considered, many novel approaches need to be explored.

\section{Further development of networked control systems-cloud control sys- tems}

Nowadays, due to its extremely powerful computation power and storage capacity, the cloud technique has been one key tool in the industry. This also brings a new opportunity to the control technique. Cloud control systems have become one of the most promising directions ${ }^{[201]}$. As the increase of the system scale, huge requirements for computation and communication cannot be ignored. In general, most of complex systems can not be controlled properly in the absence of powerful tools and adequate system information. However, the development of new technologies, including magical innovations in software and hardware, provides a necessary platform for computability. To some extent, cloud computing has come into our life. In other word, cloud computing is not only the original product concept but also a kind of service. In general, it is a byproduct of the ease-of-access to remote computing sites provided by the Internet. A new supplement, consumption, and delivery model for IT services with the foundation of Internet protocols was described in [202] . It often includes the provisioning of dynamically scalable and the virtualized resources $^{[203,204]}$. In practical systems, the cloud computing system offers a shared pool of configurable resources, which include computation, software, data access, storage services, etc. Therefore, even if without the physical location and configuration of the service provider ${ }^{[205]}$, the endusers can consume power automatically. This is to say, no matter the computers or other devices in such a system just play an utility role over the network, we can share resources, software and information, etc. For example, even if the programs are installed locally on others' computers, the users can access and use them through a web browser ${ }^{[206]}$.

The cloud control systems merge the merits of cloud computing, advanced theory of NCSs and other recent developed related results together. To some extent, it will exceed our expectation and have an extremely promising perspective. The scheme of cloud control systems is shown in Fig. 7. 


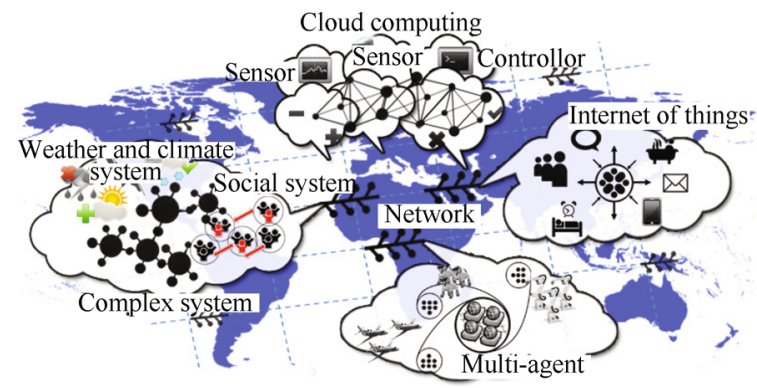

Fig. 7 Cloud control systems

In a large-scale control system, the captured information consists of information captured by ubiquitous information-sensing mobile devices, aerial sensory technologies (remote sensing), software logs, cameras, microphones, radio-frequency identification readers, and wireless sensor networks ${ }^{[207]}$. That is the origin of "Big data" [208], which is a collection of data sets. However, the set is so large and complex that on-hand traditional database management or computation tools cannot process it. As we know, the law of large numbers proposes that some useful deterministic conclusions can be obtained with the help of sufficient data. In addition, big data is confronted with a quite number of challenges including capturing, storage, search, sharing, transfer, analysis, visualization, etc. In the cloud control systems, big data will be sent to the centers of cloud computing to be processed firstly. After that, control signal, such as scheduling schemes, predictive control sequences and other useful information will be generated instantly for cloud control systems. To sum up, cloud control systems will provide us powerful tools to control the complex system, which we can not image before. More information about cloud control can be seen in [209].

\section{Conclusions}

The paper surveys some resent progresses in different components of NCSs. Quantization in NCSs, especially logarithmic and uniform quantizers, is discussed. Estimation is very important to systems with uncertainty. As an useful estimation tool, filtering, in which delay and drop-out are considered, is presented for linear and nonlinear systems, respectively. Data fusion in NCSs is significantly related to filtering. In this paper, both centralized and distributed data fusions are summarized in detail. Particularly, considering time delay and packet loss, fusion for NCSs is also discussed. In addition, some results for fault diagnosis are given. Specially, the uncertainty and the known input induced by the delay are taken into consideration. As an important control technique, predictive control is widely used for NCSs. In this paper, MPC, data packaged based predictive control and data-driven predictive control are detailedly discussed. Some progresses in distributed MPC for NCSs are also presented. In the end, cloud control systems are introduced to deal with the system, in which there exist big data and the requirement for rapid computation, and some basic concepts of the cloud control systems are given. However, there are few theoretical results for the cloud control systems. To sum up, as the vital technique of IOT, there are still fruitful results about NCSs that need to be investigated in the future.

\section{References}

[1] A. Onat, T. Naskali, E. Parlakay, O. Mutluer. Control over imperfect networks: Model-based predictive networked control systems. IEEE Transactions on Industrial Electronics, vol. 58, no. 3, pp. 905-913, 2011.

[2] Y. Ge, Q. G. Chen, M. Jiang, Y. Q. Huang. Modeling of random delays in networked control systems. Journal of Control Science and Engineering, vol. 2013, Article number 383415, 2013.

[3] L. X. Zhang, H. J. Gao, O. Kaynak. Network-induced constraints in networked control systems-A survey. IEEE Transactions on Industrial Informatics, vol. 9, no. 1, pp. 403-416, 2013.

[4] D. F. Delchamps. Stabilizing a linear system with quantized state feedback. IEEE Transactions on Automatic Control, vol. 35, no. 8, pp. 916-924, 1990.

[5] L. Bao, M. Skoglund, K. H. Johansson. Encoder-decoder design for event-triggered feedback control over bandlimited channels. In Proceedings of the American Control Conference, IEEE, Minneapolis, USA, pp. 4183-4188, 2006.

[6] S. L. Hu, D. Yue. Event-triggered control design of linear networked systems with quantizations. ISA Transactions, vol. 51, no. 1, pp. 153-162, 2012.

[7] E. Garcia, P. J. Antsaklis. Model-based event-triggered control for systems with quantization and time-varying network delays. IEEE Transactions on Automatic Control, vol.58, no. 2, pp. 422-434, 2013.

[8] L. C. Li, X. F. Wang, M. Lemmon. Stabilizing bit-rates in quantized event triggered control systems. In Proceedings of the 15th ACM International Conference on Hybrid Systems: Computation and Control, ACM, Beijing, China, pp. 245-254, 2012.

[9] N. Elia, S. K. Mitter. Stabilization of linear systems with limited information. IEEE Transactions on Automatic Control, vol. 46, no. 9, pp. 1384-1400, 2001.

[10] M. Y. Fu, L. H. Xie. The sector bound approach to quantized feedback control. IEEE Transactions on Automatic Control, vol. 50, no. 11, pp. 1698-1711, 2005.

[11] H. Ishii, T. Başar. Remote control of LTI systems over networks with state quantization. Systems \& Control Letters, vol. 54, no. 1, pp. 15-31, 2005.

[12] T. Hayakawa, H. Ishii, K. Tsumura. Adaptive quantized control for linear uncertain discrete-time systems. Automatica, vol. 45, no. 3, pp. 692-700, 2009. 
[13] T. Hayakawa, H. Ishii, K. Tsumura. Adaptive quantized control for nonlinear uncertain systems. Systems \& Control Letters, vol. 58, no. 9, pp. 625-632, 2009.

[14] K. Y. You, W. Z. Su, M. Y. Fu, L. H. Xie. Attainability of the minimum data rate for stabilization of linear systems via logarithmic quantization. Automatica, vol. 47, no. 1, pp. 170-176, 2011

[15] B. Zhou, G. R. Duan, J. Lam. On the absolute stability approach to quantized feedback control. Automatica, vol. 46 , no. 2 , pp. 337-346, 2010

[16] D. Yue, C. Peng, G. Y. Tang. Guaranteed cost control of linear systems over networks with state and input quantisations. IET Control Theory \& Applications, vol. 153, no. 6, pp. $658-664,2006$.

[17] C. Z. Zhang, G. Feng, H. J. Gao, J. B. Qiu. Generalized $H_{2}$ filter design for T-S fuzzy systems with quantization and packet loss. In Proceedings of IEEE Symposium on Computational Intelligence in Control and Automation, IEEE, Paris, France, pp. 52-59, 2011.

[18] C. Z. Zhang, G. Feng, H. J. Gao, J. B. Qiu. $H_{\infty}$ filtering for nonlinear discrete-time systems subject to quantization and packet dropouts. IEEE Transactions on Fuzzy Systems, vol. 19, no. 2, pp. 353-365, 2011.

[19] L. Li, Y. Q. Xia, J. Q. Qiu, H. J. Yang. Robust $H_{\infty}$ networked control for discrete-time fuzzy systems with state quantisation. International Journal of Systems Science, vol. 43, no. 12, pp. 2249-2260, 2012.

[20] J. J. Yan, Y. Q. Xia. Stabilisation of non-linear continuous system with input quantisation and packet dropout. IET Control Theory \& Applications, vol.6, no. 15, pp. 24262433, 2012.

[21] Y. Q. Xia, J. J. Yan, P. Shi, M. Y. Fu. Stability analysis of discrete-time systems with quantized feedback and measurements. IEEE Transactions on Industrial Informatics, vol. 9, no. 1, pp. 313-324, 2013

[22] R. W. Brockett, D. Liberzon. Quantized feedback stabilization of linear systems. IEEE Transactions on Automatic Control, vol. 45, no. 7, pp. 1279-1289, 2000.

[23] D. Liberzon. Hybrid feedback stabilization of systems with quantized signals. Automatica, vol. 39, no. 9, pp. 1543-1554, 2003.

[24] F. Fagnani, S. Zampieri. Quantized stabilization of linear systems: Complexity versus performance. IEEE Transactions on Automatic Control, vol. 49, no. 9, pp. 1534-1548, 2004

[25] F. Bullo, D. Liberzon. Quantized control via locational optimization. IEEE Transactions on Automatic Control, vol. 51, no. 1 , pp. $2-13,2006$
[26] D. Liberzon, D. Nesic. Input-to-state stabilization of linear systems with quantized state measurements. IEEE Transactions on Automatic Control, vol.52, no. 5, pp.767-781, 2007

[27] D. Nešić, D. Liberzon. A unified framework for design and analysis of networked and quantized control systems. IEEE Transactions on Automatic Control, vol. 54, no. 4, pp. 732$747,2009$.

[28] K. Y. You, L. H. Xie. Minimum data rate for mean square stabilization of discrete LTI systems over lossy channels. IEEE Transactions on Automatic Control, vol. 55, no. 10, pp. 2373-2378, 2010.

[29] K. Y. You, L. H. Xie. Minimum data rate for mean square stabilizability of linear systems with Markovian packet losses. IEEE Transactions on Automatic Control, vol. 56, no. 4, pp. 772-785, 2011

[30] E. Tian, D. Yue, X. Zhao. Quantised control design for networked control systems. IET Control Theory \& Applications, vol. 1, no. 6, pp. 1693-1699, 2007.

[31] C. De Persis. On stabilization of nonlinear systems under data rate constraints using output measurements. International Journal of Robust and Nonlinear Control, vol. 16, no. 6 , pp. 315-332, 2006.

[32] C. De Persis. Robust stabilization of nonlinear systems by quantized and ternary control. Systems \& Control Letters, vol. 58, no. 8, pp. 602-608, 2009 .

[33] J. J. Yan, Y. Q. Xia, B. Liu, M. Y. Fu. Stabilisation of quantised linear systems with packet dropout. IET Control Theory \& Applications, vol. 5, no. 8, pp. 982-989, 2011.

[34] J. J. Yan, Y. Q. Xia, L. Li. Stabilization of fuzzy systems with quantization and packet dropout. International Journal of Robust and Nonlinear Control, vol. 24, no. 10, pp. 1563-1583, 2014.

[35] Y. Q. Xia, J. J. Yan, J. Z. Shang, M. Y. Fu, B. Liu. Stabilization of quantized systems based on Kalman filter. Control Engineering Practice, vol. 20, no. 10, pp. 954-962, 2012.

[36] Y. Sharon, D. Liberzon. Input to state stabilizing controller for systems with coarse quantization. IEEE Transactions on Automatic Control, vol. 57, no. 4, pp. 830-844, 2012.

[37] R. E. Kalman. A new approach to linear filtering and prediction problems. Journal of Fluids Engineering, vol. 82, no. 1 , pp. 35-45, 1960.

[38] K. Y. You, L. H. Xie. Kalman filtering with scheduled measurements. IEEE Transactions on Signal Processing, vol. 61, no. 6 , pp. $1520-1530,2013$ 
[39] M. Sahebsara, T. W. Chen, S. L. Shah. Optimal $H_{2}$ filtering in networked control systems with multiple packet dropout. IEEE Transactions on Automatic Control, vol. 52, no. 8, pp. $1508-1513,2007$.

[40] M. Sahebsara, T. W. Chen, S. L. Shah. Optimal $H_{\infty}$ filtering in networked control systems with multiple packet dropouts. Systems \& Control Letters, vol. 57, no. 9, pp. 696$702,2008$.

[41] W. A. Zhang, L. Yu, H. B. Song. $H_{\infty}$ filtering of networked discrete-time systems with random packet losses. Information Sciences, vol. 179, no. 22, pp. 3944-3955, 2009.

[42] B. Sinopoli, L. Schenato, M. Franceschetti, K. Poolla, M. I. Jordan, S. S. Sastry. Kalman filtering with intermittent observations. IEEE Transactions on Automatic Control, vol. 49, no. 9, pp. 1453-1464, 2004.

[43] X. He, Z. D. Wang, D. Zhou. Robust $H_{\infty}$ filtering for networked systems with multiple state delays. International Journal of Control, vol. 80, no. 8, pp. 1217-1232, 2007.

[44] S. L. Hu, D. Yue. Event-based $H_{\infty}$ filtering for networked system with communication delay. Signal Processing, vol. 92, no. 9, pp. 2029-2039, 2012.

[45] M. Moayedi, Y. K. Foo, Y. C. Soh. Adaptive Kalman filtering in networked systems with random sensor delays, multiple packet dropouts and missing measurements. IEEE Transactions on Signal Processing, vol. 58, no. 3, pp. 15771588,2010

[46] L. Schenato. Optimal estimation in networked control systems subject to random delay and packet drop. IEEE Transactions on Automatic Control, vol. 53, no. 5, pp. 1311-1317, 2008.

[47] M. Sahebsara, T. Chen, S. L. Shah. Optimal filtering with random sensor delay, multiple packet dropout and uncertain observations. International Journal of Control, vol. 80, no. 2, pp. 292-301, 2007.

[48] G. L. Wei, Z. D. Wang, X. He, H. S. Shu. Filtering for networked stochastic time-delay systems with sector nonlinearity. IEEE Transactions on Circuits and Systems II: Express Briefs, vol. 56, no. 1, pp. 71-75, 2009.

[49] Y. Shi, H. Z. Fang. Kalman filter-based identification for systems with randomly missing measurements in a network environment. International Journal of Control, vol. 83, no. 3, pp. 538-551, 2010.

[50] M. Moayedi, Y. K. Foo, Y. C. Soh. Filtering for networked control systems with single/multiple measurement packets subject to multiple-step measurement delays and multiple packet dropouts. International Journal of Systems Science, vol. 42, no. 3, pp. 335-348, 2011.
[51] D. Yue, Q. L. Han. Network-based robust $H_{\infty}$ filtering for uncertain linear systems. IEEE Transactions on Signal Processing, vol. 54, no. 11, pp. 4293-4301, 2006.

[52] Y. Q. Xia, J. Q. Han. Robust Kalman filtering for systems under norm bounded uncertainties in all system matrices and error covariance constraints. Journal of Systems Science and Complexity, vol. 18, no. 4, pp. 439-445, 2005.

[53] H. J. Yang, Y. Q. Xia, P. Shi, M. Y. Fu. A novel delta operator Kalman filter design and convergence analysis. IEEE Transactions on Circuits and Systems I: Regular Papers, vol. 58, no. 10, pp. 2458-2468, 2011.

[54] W. Xie, Y. Q. Xia. Data-driven method for Kalman filtering. In Proceedings of the 2nd International Conference on Intelligent Control and Information Processing, IEEE, Harbin, China, pp. 830-835, 2011.

[55] J. F. Wu, Q. S. Jia, K. H. Johansson, L. Shi. Event-based sensor data scheduling: Trade-off between communication rate and estimation quality. IEEE Transactions on Automatic Control, vol. 58, no. 4, pp. 1041-1046, 2013.

[56] D. E. Quevedo, D. Nešić. Robust stability of packetized predictive control of nonlinear systems with disturbances and Markovian packet losses. Automatica, vol. 48, no. 8, pp. 1803-1811, 2012.

[57] R. N. Yang, P. Shi, G. P. Liu. Filtering for discrete-time networked nonlinear systems with mixed random delays and packet dropouts. IEEE Transactions on Automatic Control, vol. 56, no. 11, pp. 2655-2660, 2011.

[58] H. L. Dong, Z. D. Wang, H. J. Gao. Robust $H_{\infty}$ filtering for a class of nonlinear networked systems with multiple stochastic communication delays and packet dropouts. IEEE Transactions on Signal Processing, vol. 58, no. 4, pp. 1957-1966, 2010.

[59] M. Hilairet, F. Auger, E. Berthelot. Speed and rotor flux estimation of induction machines using a two-stage extended Kalman filter. Automatica, vol. 45, no. 8, pp. 1819-1827, 2009 .

[60] H. W. Sorenson. Kalman Filtering: Theory and Application, Piscataway, New Jersey, USA: IEEE, 1985.

[61] J. J. LaViola. A comparison of unscented and extended Kalman filtering for estimating quaternion motion. In Proceedings of the American Control Conference, IEEE, Colorado, USA, pp. 2435-2440, 2003.

[62] N. J. Gordon, D. J. Salmond, A. F. M. Smith. Novel approach to nonlinear/non-Gaussian Bayesian state estimation. IEE Proceedings F on Radar and Signal Processing, vol. 140, no. 2, pp. 107-113, 1993.

[63] S. Guadarrama, A. Ruiz-Mayor. Approximate robotic mapping from sonar data by modeling perceptions with antonyms. Information Sciences, vol. 180, no. 21, pp. 4164$4188,2010$. 
[64] H. P. Liu, F. C. Sun. Efficient visual tracking using particle filter with incremental likelihood calculation. Information Sciences, vol. 195, pp. 141-153, 2012.

[65] Y. Q. Xia, Z. H. Deng, L. Li, X. M. Geng. A new continuousdiscrete particle filter for continuous-discrete nonlinear systems. Information Sciences, vol. 242, pp. 64-75, 2013.

[66] S. Kluge, K. Reif, M. Brokate. Stochastic stability of the extended Kalman filter with intermittent observations. IEEE Transactions on Automatic Control, vol. 55, no. 2, pp. 514$518,2010$.

[67] L. Li, Y. Q. Xia. Stochastic stability of the unscented Kalman filter with intermittent observations. Automatica, vol. 48, no. 5, pp. 978-981, 2012.

[68] L. Li, Y. Q. Xia. Unscented Kalman filter over unreliable communication networks with Markovian packet dropouts. IEEE Transactions on Automatic Control, vol.58, no. 12, pp. 3224-3230, 2013.

[69] R. A. Singer, A. J. Kanyuck. Computer control of multiple site track correlation. Automatica, vol. 7, no. 4, pp. 455-463, 1971.

[70] D. Willner, C. B. Chang, K. P. Dunn. Kalman filter algorithms for a multi-sensor system. In Proceedings of IEEE Conference on Decision and Control including the 15th Symposium on Adaptive Processes, IEEE, Clearwater, USA, pp. 570-574, 1976

[71] K. Salahshoor, M. Mosallaei, M. Bayat. Centralized and decentralized process and sensor fault monitoring using data fusion based on adaptive extended Kalman filter algorithm. Measurement, vol. 41, no. 10, pp. 1059-1076, 2008.

[72] M. Munz, M. Mählisch, K. Dietmayer. Generic centralized multi sensor data fusion based on probabilistic sensor and environment models for driver assistance systems. IEEE Intelligent Transportation Systems Magazine, vol. 2, no. 1, pp. 6-17, 2010.

[73] A. Polychronopoulos, U. Scheunert, F. Tango. Centralized data fusion for obstacle and road borders tracking in a collision warning system. In Proceedings of the 7th International Conference on Information Fusion, 2004

[74] G. J. Bierman, M. R. Belzer. A decentralized square root information filter/smoother. In Proceedings of the 24th IEEE Conference on Decision and Control, IEEE, Fort Lauderdale, USA, pp. 1902-1905, 1985.

[75] N. A. Carlson. Federated square root filter for decentralized parallel processors. IEEE Transactions on Aerospace and Electronic Systems, vol. 26, no. 3, pp. 517-525, 1990.

[76] D. A. Castanon, D. Teneketzis. Distributed estimation algorithms for nonlinear systems. IEEE Transactions on $\mathrm{Au}$ tomatic Control, vol. 30, no. 5, pp. 418-425, 1985.
[77] R. Lobbia, M. Kent. Data fusion of decentralized local tracker outputs. IEEE Transactions on Aerospace and Electronic Systems, vol. 30, no. 3, pp. 787-799, 1994.

[78] Y. Bar-Shalom. On the track-to-track correlation problem. IEEE Transactions on Automatic Control, vol.26, no. 2 pp. 571-572, 1981

[79] S. Grime, H. F. Durrant-Whyte, P. Ho. Communication in decentralized data-fusion systems. In Proceedings of the American Control Conference, IEEE, Chicago, USA, pp. 3299-3303, 1992.

[80] R. Kumar, M. Wolenetz, B. Agarwalla, J. S. Shin, P. W. Hutto, A. Paul, U. Ramachandran. DFuse: A framework for distributed data fusion. In Proceedings of the 1st International Conference on Embedded Networked Sensor Systems, ACM, New York, USA, pp. 114-125, 2003.

[81] M. S. Mahmoud, Y. Q. Xia. Networked Filtering and Fusion in Wireless Sensor Networks, New York: CRC Press, 2014 .

[82] S. Challa, M. Palaniswami, A. Shilton. Distributed data fusion using support vector machines. In Proceedings of the 5th International Conference on Information Fusion, IEEE, Annapolis, USA, pp. 881-885, 2002.

[83] C. S. Regazzoni, A. Tesei. Distributed data fusion for realtime crowding estimation. Signal Processing, vol. 53, no. 1, pp. 47-63, 1996.

[84] M. E. Liggins, C. Y. Chong, I. Kadar, M. G. Alford, V. Vannicola, S. Thomopoulos. Distributed fusion architectures and algorithms for target tracking. Proceedings of the IEEE, vol. 85, no. 1, pp. 95-107, 1997.

[85] E. Besada-Portas, J. A. Lopez-Orozco, J. Besada, J. M. De la Cruz. Multisensor fusion for linear control systems with asynchronous, out-of-sequence and erroneous data. Automatica, vol. 47, no. 7, pp. 1399-1408, 2011.

[86] X. J. Shen, E. B. Song, Y. M. Zhu, Y. T. Luo. Globally optimal distributed Kalman fusion with local out-of-sequencemeasurement updates. IEEE Transactions on Automatic Control, vol. 54, no. 8, pp. 1928-1934, 2009.

[87] B. Chen, W. A. Zhang, L. Yu. Distributed fusion estimation with missing measurements, random transmission delays and packet dropouts. IEEE Transactions on Automatic Control, vol. 59, no. 7, pp. 1961-1967, 2014.

[88] Y. Q. Xia, J. Z. Shang, J. Chen, G. P. Liu. Data fusion over network. In Proceedings of the 27th Chinese Control Conference, IEEE, Kunming, China, pp. 452-456, 2008.

[89] Y. Q. Xia, J. Z. Shang, J. Chen, G. P. Liu. Networked data fusion with packet losses and variable delays. IEEE Transactions on Systems, Man, and Cybernetics - Part B: Cybernetics, vol. 39, no. 5, pp. 1107-1120, 2009. 
[90] C. Zhu, Y. Q. Xia, L. P. Yan, M. Y. Fu. Multi-channel networked data fusion with intermittent observations. In Proceedings of the 29th Chinese Control Conference, IEEE, Beijing, China, pp. 4317-4322, 2010.

[91] C. Zhu, Y. Q. Xia, L. P. Yan, M. Y. Fu. Centralised fusion over unreliable networks. International Journal of Control, vol. 85, no. 4, pp. 409-418, 2012.

[92] W. A. Zhang, G. Feng, L. Yu. Multi-rate distributed fusion estimation for sensor networks with packet losses. Automatica, vol. 48, no. 9, pp. 2016-2028, 2012.

[93] Y. Liang, T. W. Chen, Q. Pan. Multi-rate stochastic $H_{\infty}$ filtering for networked multi-sensor fusion. Automatica, vol. 46, no. 2, pp. 437-444, 2010.

[94] L. P. Yan, B. Xiao, Y. Q. Xia, M. Y. Fu. State estimation for asynchronous multirate multisensor nonlinear dynamic systems with missing measurements. International Journal of Adaptive Control and Signal Processing, vol. 26, no. 6, pp. 516-529, 2012.

[95] M. Renzo, L. Imbriglio, F. Graziosi, F. Santucci. Distributed data fusion over correlated log-normal sensing and reporting channels: Application to cognitive radio networks. IEEE Transactions on Wireless Communications, vol. 8, no. 12, pp. 5813-5821, 2009.

[96] L. P. Yan, X. R. Li, Y. Q. Xia, M. Y. Fu. Optimal sequential and distributed fusion for state estimation in crosscorrelated noise. Automatica, vol. 49, no. 12, pp. 3607-3612, 2013.

[97] X. L. Bian, Y. Q. Xia, Z. H. Deng, M. Y. Fu. Onechannel networked data fusion with communication constraint. Journal of the Franklin Institute, vol. 351, no. 1, pp. 156-173, 2014.

[98] X. L. Bian, Y. Q. Xia. Energy efficient data fusion over wireless channels with power control. IET Signal Processing, vol. 9, no. 3, pp. 206-217, 2015.

[99] H. Ye, S. X. Ding. Fault detection of networked control systems with network-induced delay. In Proceedings of the 8th International Conference on Control, Automation, Robotics and Vision, IEEE, Kunming, China, pp. 294-297, 2004.

[100] W. Zhang, M. S. Branicky, S. M. Phillips. Stability of networked control systems. IEEE Control Systems Magazine, vol. 21, no. 1, pp. 84-99, 2001.

[101] H. Ye, P. Zhang, S. X. Ding, G. Z. Wang. A time-frequency domain fault detection approach based on parity relation and wavelet transform. In Proceedings of the 39th IEEE Conference on Control and Decision, IEEE, Sydney, Australia, pp. 4156-4161, 2000.

[102] D. Huang, S. K. Nguang. Robust fault estimator design for uncertain networked control systems with random time delays: An ILMI approach. Information Sciences, vol. 180, no. 3, pp. 465-480, 2010.
[103] L. B. Xie, H. J. Fang, Y. Zheng. Guaranteed cost control for networked control systems. Journal of Control Theory and Applications, vol. 2, no. 2, pp. 143-148, 2004.

[104] H. Ye, Y. Q. Wang. Application of parity relation and stationary wavelet transform to fault detection of networked control systems. In Proceedings of the 1st IEEE Conference on Industrial Electronics and Application, IEEE, Singapore, pp. 1-6, 2006.

[105] H. Ye, G. Z. Wang, S. X. Ding. A new parity space approach for fault detection based on stationary wavelet transform. IEEE Transactions on Automatic Control, vol. 49, no. 2, pp. 281-287, 2004.

[106] H. Ye, R. He, H. Liu, G. Z. Wang. A new approach for fault detection of networked control systems. In Proceedings of the 14th IFAC Symposium on System Identification, IFAC, New castle, Australia, pp. 654-659, 2006.

[107] Z. H. Huo, H. J. Fang. Robust $H_{\infty}$ filter design for networked control system with random time delays. In Proceedings of the 10th IEEE International Conference on Engineering of Complex Computer Systems, IEEE, Shanghai, China, pp. 333-340, 2005.

[108] Z. H. Huo, H. J. Fang. Research on robust fault detection for NCS with long delay based on LMI. International Journal of Advances in Systems Science and Applications, vol. 5, no. 3, pp. 404-410, 2005.

[109] Z. H. Huo, H. J. Fang. Fault-tolerant control research for networked control system under communication constraints. Acta Automatica Sinica, vol. 32, no. 5, pp. 659-666, 2006

[110] Z. H. Huo, H. J. Fang. Fault-tolerant control of networked control systems with random time-delays. Information and Control, vol. 35, no. 5, pp. 584-587, 2006.

[111] H. J. Fang, H. Zhang, Y. W. Fang, F. Yang. Quasi T-S fuzzy models and stable controllers for networked control systems. In Proceedings of the 6th World Congress on Intelligent Control and Automation, IEEE, Dalian, China, pp. 220-223, 2006.

[112] Y. Zheng, H. J. Fang, H. O. Wang. Takagi-sugeno fuzzymodel-based fault detection for networked control systems with Markov delays. IEEE Transactions on Systems, Man, and Cybernetics - Part B: Cybernetics, vol. 36, no. 4, pp. 924-929, 2006.

[113] H. J. Fang, F. Yang, Y. Zheng, H. Zhang. Fuzzy modeling and fault detection for networked control systems. In Proceedings of the 6th IFAC Symposium on Fault Detection, Supervision and Safety of Technical Processes, IFAC, Beijing, China, pp. 1091-1096, 2006. 
[114] Z. H. Mao, B. Jiang, P. Shi. Protocol and fault detection design for nonlinear networked control systems. IEEE Transactions on Circuits and Systems II: Express Briefs, vol. 56, no. 3, pp. 255-259, 2009.

[115] B. Liu, Y. Q. Xia, Y. Yang, M. Y. Fu. Robust fault detection of linear systems over networks with bounded packet loss. Journal of the Franklin Institute, vol. 349, no. 7, pp. 2480-2499, 2012.

[116] H. J. Yang, Y. Q. Xia, B. Liu. Fault detection for TCS fuzzy discrete systems in finite-frequency domain. IEEE Transactions on Systems, Man, and Cybernetics - Part B: Cybernetics, vol. 41, no. 4, pp. 911-920, 2011.

[117] B. Liu, Y. Q. Xia. Fault detection and compensation for linear systems over networks with random delays and clock asynchronism. IEEE Transactions on Industrial Electronics, vol. 58, no. 9, pp. 4396-4406, 2011.

[118] Y. Q. Xia, B. Liu. Maximum likelihood ratio detection of abrupt state change for MIMO linear systems based on frequency domain data. International Journal of Robust and Nonlinear Control, vol. 23, no. 8, pp. 858-877, 2013.

[119] Y. Q. Xia, A. Amann, B. Liu. Detection of abrupt changes in electrocardiogram with generalised likelihood ratio algorithm. IET Signal Processing, vol. 4, no.6, pp.650-657, 2010 .

[120] M. Y. Chow, Y. Tipsuwan. Network-based control systems: A tutorial. In Proceedings of the 27th Annual Conference of the IEEE Industrial Electronics Society, IEEE, Denver, USA, pp. 1593-1602, 2001.

[121] D. Q. Mayne, J. B. Rawlings, C. V. Rao, P. O. M. Scokaert. Constrained model predictive control: Stability and optimality. Automatica, vol. 36, no. 6, pp. 789-814, 2000

[122] H. Chen, F. Allgöwer. A quasi-infinite horizon nonlinear model predictive control scheme with guaranteed stability. Automatica, vol. 34, no. 10, pp. 1205-1217, 1998.

[123] G. W. Irwin, J. Chen, A. McKernan, W. G. Scanlon. Codesign of predictive controllers for wireless network control. IET Control Theory \& Applications, vol. 4, no. 2, pp. 186196, 2010.

[124] G. P. Liu, J. X. Mu, D. Rees, S. C. Chai. Design and stability analysis of networked control systems with random communication time delay using the modified MPC. International Journal of Control, vol. 79, no. 4, pp. 288-297, 2006.

[125] H. Zhang, Y. Shi, M. X. Liu. $H_{\infty}$ step tracking control for networked discrete-time nonlinear systems with integral and predictive actions. IEEE Transactions on Industrial Informatics, vol. 9, no. 1, pp. 337-345, 2013.
[126] Y. B. Zhao, G. P. Liu, D. Rees. Improved predictive control approach to networked control systems. IET Control Theory \& Applications, vol. 2, no. 8, pp. 675-681, 2008.

[127] Y. B. Zhao, G. P. Liu, D. Rees. Integrated predictive control and scheduling co-design for networked control systems. IET Control Theory \& Applications, vol. 2, no. 1, pp. 7-15, 2008

[128] G. F. Zhang, X. Chen, T. W. Chen. A model predictive control approach to networked systems. In Proceedings of the 46th IEEE Conference on Decision and Control, IEEE, New Orleans, USA, pp. 3339-3344, 2007.

[129] G. C. Goodwin, H. Haimovich, D. E. Quevedo, J. S. Welsh. A moving horizon approach to networked control system design. IEEE Transactions on Automatic Control, vol. 49, no. 9 , pp. 1427-1445, 2004.

[130] P. Varutti, B. Kern, T. Faulwasser, R. Findeisen. Eventbased model predictive control for networked control systems. In Proceedings of the 28th Chinese Control Conference and Decision and the 48th IEEE Conference on Control Conference, IEEE, Shanghai, China, pp. 567-572, 2009.

[131] D. E. Quevedo, D. Nešić. Input-to-state stability of packetized predictive control over unreliable networks affected by packet-dropouts. IEEE Transactions on Automatic Control, vol. 56, no. 2, pp. 370-375, 2011

[132] D. Muñoz de la Peña, P. D. Christofides. Lyapunovbased model predictive control of nonlinear systems subject to data losses. IEEE Transactions on Automatic Control, vol. 53, no. 9, pp. 2076-2089, 2008.

[133] G. Pin, T. Parisini. Networked predictive control of uncertain constrained nonlinear systems: Recursive feasibility and input-to-state stability analysis. IEEE Transactions on Automatic Control, vol. 56, no. 1, pp. 72-87, 2011.

[134] Y. Q. Xia, G. P. Liu, P. Shi, J. Chen, D. Rees. Robust constrained model predictive control based on parameterdependent Lyapunov functions. Circuits, Systems and Signal Processing, vol. 27, no. 4, pp. 429-446, 2008.

[135] Y. Iino, T. Hatanaka, M. Fujita. Event-predictive control for energy saving of wireless networked control system. In Proceedings of the American Control Conference, IEEE, Saint Louis, USA, pp. 2236-2242, 2009.

[136] E. Garcia, P. J. Antsaklis. Model-based event-triggered control with time-varying network delays. Proceedings of the 50th IEEE Conference on Decision and Control and European Control Conference, IEEE, Orlando, USA, pp. 16501655, 2011.

[137] A. Eqtami, D. V. Dimarogonas, K. J. Kyriakopoulos. Novel event-triggered strategies for model predictive controllers. In Proceedings of the 50th IEEE Conference on Decision and Control and European Control Conference, IEEE, Orlando, USA, pp. 3392-3397, 2011. 
[138] E. Henriksson, D. E. Quevedo, H. Sandberg, K. H. Johansson. Self-triggered model predictive control for network scheduling and control. In Proceedings of the 8th IFAC Symposium on Advanced Control of Chemical Processes, IFAC, Furama Riverfront, Singapore, pp. 432-438, 2012.

[139] J. Wu, L. Q. Zhang, T. W. Chen. Model predictive control for networked control systems. International Journal of Robust and Nonlinear Control, vol. 19, no.9, pp. 1016-1035, 2009.

[140] J. F. Liu, D. Muñoz de la Peña, P. D. Christofides, J. F. Davis. Lyapunov-based model predictive control of nonlinear systems subject to time-varying measurement delays. International Journal of Adaptive Control and Signal Processing, vol. 23, no. 8, pp. 788-807, 2009.

[141] L. C. Jin, R. Kumar, N. Elia. Model predictive controlbased real-time power system protection schemes. IEEE Transactions on Power Systems, vol. 25, no. 2, pp. 988-998, 2010.

[142] B. Yu, Y. Shi, J. Huang. Modified generalized predictive control of networked systems with application to a hydraulic position control system. Journal of Dynamic Systems, Measurement, and Control, vol. 133, no. 3, Article number 031009, 2011.

[143] Y. Zou, T. Chen, S. Li. Network-based predictive control of multirate systems. IET Control Theory \& Applications, vol. 4, no. 7, pp. 1145-1156, 2010.

[144] Y. Zhang, S. Y. Li. Networked model predictive control based on neighbourhood optimization for serially connected large-scale processes. Journal of Process Control, vol. 17, no. 1 , pp. 37-50, 2007.

[145] G. P. Liu, J. X. Mu, D. Rees. Networked predictive control of systems with random communication delay. In Proceedings of UKACC International Conference on Control, Bath, UK, 2004.

[146] Y. B. Zhao, G. P. Liu, D. Rees. Networked predictive control systems based on the Hammerstein model. IEEE Transactions on Circuits and Systems II: Express Briefs, vol. 55, no. 5, pp. 469-473, 2008.

[147] D. E. Quevedo, J. Ostergaard, D. Nešić. Packetized predictive control of stochastic systems over bit-rate limited channels with packet loss. IEEE Transactions on Automatic Control, vol. 56, no. 12, pp. 2854-2868, 2011.

[148] D. E. Quevedo, E. I. Silva, G. C. Goodwin. Packetized predictive control over erasure channels. In Proceedings of the American Control Conference, IEEE, New York, USA, pp. 1003-1008, 2007.

[149] P. Mhaskar, N. H. El-Farra, P. D. Christofides. Predictive control of switched nonlinear systems with scheduled mode transitions. IEEE Transactions on Automatic Control, vol. 50, no. 11, pp. 1670-1680, 2005.
[150] B. Xue, N. Li, S. Li, Q. Zhu. Robust model predictive control for networked control systems with quantisation. IET Control Theory \& Applications, vol.4, no. 12, pp. 28962906, 2010.

[151] D. Srinivasagupta, H. Schättler, B. Joseph. Time-stamped model predictive control: An algorithm for control of processes with random delays. Computers \& Chemical Engineering, vol. 28, no. 8, pp. 1337-1346, 2004.

[152] Y. Q. Xia, H. J. Yang, P. Shi, M. Y. Fu. Constrained infinite-horizon model predictive control for fuzzy-discretetime systems. IEEE Transactions on Fuzzy Systems, vol. 18, no. 2, pp. 429-436, 2010.

[153] Y. Q. Xia, M. Y. Fu, P. Shi. Analysis and Synthesis of Dynamical Systems with Time-delays, Berlin, Germany, Heidelberg: Springer, 2009.

[154] Y. Q. Xia, M. Y. Fu, G. P. Liu. Analysis and Synthesis of Networked Control Systems, Berlin, Germany, Heidelberg: Springer, 2011.

[155] Y. Q. Xia, G. P. Liu, M. Y. Fu, D. Rees. Predictive control of networked systems with random delay and data dropout. IET Control Theory \& Applications, vol. 3, no. 11, pp. 1476-1486, 2009.

[156] Y. Q. Xia, F. M. Fu, B. Liu, G. P. Liu. Design and performance analysis of networked control systems with random delay. Journal of Systems Engineering and Electronics, vol. 20, no. 4, pp. 807-822, 2009.

[157] S. C. Chai, G. P. Liu, D. Rees. Design and implementation of networked predictive control systems. In Proceedings of the 16th IFAC World Congress, IFAC, Prague, Czech Republic, 2005.

[158] W. S. Hu, G. P. Liu, D. Rees. Design and implementation of networked predictive control systems based on round trip time delay measurement. In Proceedings of the American Control Conference, IEEE, Minneapolis, USA, pp. 674-679, 2006.

[159] S. C. Chai, G. P. Liu, D. Rees, Y. Q. Xia. Design and practical implementation of internet-based predictive control of a servo system. IEEE Transactions on Control Systems Technology, vol. 16, no. 1, pp. 158-168, 2008.

[160] G. P. Liu, D. Rees, S. C. Chai. Design and practical implementation of networked predictive control systems. In Proceedings of the 12th IEEE International Conference on Networking, Sensing and Control, IEEE, Tucson, USA, pp. 336-341, 2005.

[161] G. P. Liu, Y. Q. Xia, D. Rees, W. S. Hu. Design and stability criteria of networked predictive control systems with random network delay in the feedback channel. IEEE Transactions on Systems, Man, and Cybernetics - Part C: Applications and Reviews, vol. 37, no. 2, pp. 173-184, 2007. 
[162] W. S. Hu, G. P. Liu, D. Rees. Event-driven networked predictive control. IEEE Transactions on Industrial Electronics, vol. 54, no. 3, pp. 1603-1613, 2007.

[163] Y. Q. Xia, L. Li, G. P. Liu, P. Shi. $H_{\infty}$ predictive control of networked control systems. International Journal of Control, vol. 84, no. 6, pp. 1080-1097, 2011.

[164] R. Wang, G. P. Liu, W. Wang, D. Rees, Y. B. Zhao. $H_{\infty}$ control for networked predictive control systems based on the switched Lyapunov function method. IEEE Transactions on Industrial Electronics, vol. 57, no. 10, pp. 3565$3571,2010$.

[165] B. Liu, Y. Q. Xia, M. S. Mahmoud, H. Wu, S. S. Cui. New predictive control scheme for networked control systems. Circuits, Systems, and Signal Processing, vol. 31, no. 3, pp. 945-960, 2012.

[166] G. P. Liu, S. C. Chai, J. X. Mu, D. Rees. Networked predictive control of systems with random delay in signal transmission channels. International Journal of Systems Science, vol. 39, no. 11, pp. 1055-1064, 2008.

[167] G. P. Liu, Y. Q. Xia, J. Chen, D. Rees, W. S. Hu. Networked predictive control of systems with random network delays in both forward and feedback channels. IEEE Transactions on Industrial Electronics, vol. 54, no. 3, pp. 12821297, 2007.

[168] G. P. Liu. Predictive controller design of networked systems with communication delays and data loss. IEEE Transactions on Circuits and Systems II: Express Briefs, vol. 57, no. 6, pp. 481-485, 2010.

[169] R. N. Yang, G. P. Liu, P. Shi, C. Thomas, M. V. Basin. Predictive output feedback control for networked control systems. IEEE Transactions on Industrial Electronics, vol. 61, no. 1, pp. 512-520, 2014.

[170] Y. Q. Xia, J. Chen, G. P. Liu, D. Rees. Stability analysis of networked predictive control systems with random network delay. In Proceedings of IEEE International Conference on Networking, Sensing and Control, IEEE, London, UK, pp. 815-820, 2007.

[171] R. Wang, B. Wang, G. P. Liu, W. Wang, D. Rees. $H_{\infty}$ controller design for networked predictive control systems based on the average dwell-time approach. IEEE Transactions on Circuits and Systems II: Express Briefs, vol. 57, no. 4, pp. 310-314, 2010.

[172] R. Kadali, B. Huang, A. Rossiter. A data driven subspace approach to predictive controller design. Control Engineering Practice, vol. 11, no. 3, pp. 261-278, 2003.

[173] D. Laurí, J. A. Rossiter, J. Sanchis, M. Martínez. Datadriven latent-variable model-based predictive control for continuous processes. Journal of Process Control, vol. 20, no. 10 , pp. 1207-1219, 2010.
[174] J. X. Xu, Z. S. Hou. Notes on data-driven system approaches. Acta Automatica Sinica, vol. 35, no. 6, pp. 668$675,2009$.

[175] Y. Q. Xia, W. Xie, B. Liu, X. Y. Wang. Data-driven predictive control for networked control systems. Information Sciences, vol. 235, pp. 45-54, 2013.

[176] G. Chaloulos, P. Hokayem, J. Lygeros. Distributed hierarchical MPC for conflict resolution in air traffic control. In Proceedings of the American Control Conference, IEEE, Baltimore, USA, pp. 3945-3950, 2010.

[177] T. Keviczky, F. Borrelli, K. Fregene, D. Godbole, G. J. Balas. Decentralized receding horizon control and coordination of autonomous vehicle formations. IEEE Transactions on Control Systems Technology, vol.16, no. 1, pp.19-33, 2008.

[178] B. T. Stewart, S. J. Wright, J. B. Rawlings. Cooperative distributed model predictive control for nonlinear systems. Journal of Process Control, vol. 21, no. 5, pp. 698-704, 2011.

[179] A. N. Venkat, I. A. Hiskens, J. B. Rawlings, S. J. Wright. Distributed MPC strategies with application to power system automatic generation control. IEEE Transactions on Control Systems Technology, vol. 16, no. 6, pp. 1192-1206, 2008.

[180] Y. Kuwata, A. Richards, T. Schouwenaars, J. P. How. Distributed robust receding horizon control for multivehicle guidance. IEEE Transactions on Control Systems Technology, vol. 15, no. 4, pp.627-641, 2007.

[181] P. Trodden, A. Richards. Cooperative distributed MPC of linear systems with coupled constraints. Automatica, vol. 49, no. 2, pp. 479-487, 2013.

[182] A. Richards, J. P. How. Robust distributed model predictive control. International Journal of Control, vol. 80, no. 9 , pp. 1517-1531, 2007.

[183] M. A. Müller, M. Reble, F. Allgöwer. A general distributed MPC framework for cooperative control. In Proceedings of the 18th IFAC World Congress, IFAC, Milano, Italy, pp. 7987-7992, 2011.

[184] P. Wang, B. C. Ding. Distributed RHC for tracking and formation of nonholonomic multi-vehicle systems. IEEE Transactions on Automatic Control, vol. 59, no. 6, pp. 1439$1453,2014$.

[185] W. B. Dunbar. Distributed receding horizon control of dynamically coupled nonlinear systems. IEEE Transactions on Automatic Control, vol. 52, no. 7, pp. 1249-1263, 2007.

[186] J. M. Maestre, R. R. Negenborn. Distributed Model Predictive Control Made Easy, The Netherlands: Springer, 2014 
[187] R. R. Negenborn, J. M. Maestre. Distributed model predictive control: An overview and roadmap of future research opportunities. IEEE Control Systems Magazine, vol. 34, no. 4, pp. 87-97, 2014.

[188] A. Eqtami, D. V. Dimarogonas, K. J. Kyriakopoulos. Event-based model predictive control for the cooperation of distributed agents. In Proceedings of the American Control Conference, IEEE, Montreal, Canada, pp. 6473-6478, 2012.

[189] A. Eqtami, D. V. Dimarogonas, K. J. Kyriakopoulos. Event-triggered strategies for decentralized model predictive controllers. In Proceedings of the 18th IFAC World Congress, IFAC, Milano, Italy, pp. 10068-10073, 2011.

[190] W. B. Dunbar. Distributed Receding Horizon Control of Multiagent Systems, Ph. D dissertation, California Institute of Technology, USA, 2004.

[191] W. B. Dunbar, R. M. Murray. Distributed receding horizon control for multi-vehicle formation stabilization. Automatica, vol. 42, no. 4, pp. 549-558. 2006.

[192] T. Keviczky, F. Borrelli, G. J. Balas. Decentralized receding horizon control for large scale dynamically decoupled systems. Automatica, vol. 42, no. 12, pp. 2105-2115, 2006.

[193] H. P. Li, Y. Shi. Distributed model predictive control of constrained nonlinear systems with communication delays. Systems \& Control Letters, vol.62, no. 10, pp. 819-826, 2013.

[194] H. P. Li, Y. Shi. Robust distributed model predictive control of constrained continuous-time nonlinear systems: A robustness constraint approach. IEEE Transactions on Automatic Control, vol. 59, no. 6, pp. 1673-1678, 2014.

[195] H. P. Li, Y. Shi. Distributed receding horizon control of large-scale nonlinear systems: Handling communication delays and disturbances. Automatica, vol. 50, no. 4, pp. 1264 $1271,2014$.

[196] Y. Q. Xia, M. Y. Fu. Compound Control Methodology for Flight Vehicles, Berlin, Germany, Heidelberg: Springer, 2013.

[197] N. Zhou, Y. Q. Xia, K. F. Lu, Y. Li. Decentralised finitetime attitude synchronisation and tracking control for rigid spacecraft. International Journal of Systems Science, to be published.

[198] N. Zhou, Y. Q. Xia, M. L. Wang, M. Y. Fu. Finite-time attitude control of multiple rigid spacecraft using terminal sliding mode. International Journal of Robust and Nonlinear Control, to be published.

[199] N. Zhou, Y. Q. Xia, K. F. Lu. Attitude synchronization of rigid spacecraft using terminal sliding mode. In Proceedings of the 32nd Chinese Control Conference, IEEE, Xi'an, China, pp. 706-711, 2013.
[200] Y. Q. Xia, N. Zhou, K. F. Lu, Y. Li. Attitude control of multiple rigid bodies with uncertainties and disturbances. IEEE/CAA Journal of Automatica Sinica, vol. 2, no. 1, pp. $2-10,2015$.

[201] Y. Q. Xia. From networked control systems to cloud control systems. In Proceedings of the 31st Chinese Control Conference, IEEE, Hefei, China, pp. 5878-5883, 2012.

[202] The economist, cloud computing: Clash of the clouds, [Online], Available: http://www.economist.com/node/14637206, 3 November, 2009.

[203] Gartner says cloud computing will be as influential as e-business, [Online], Availabe: http://www.gartner.com/newsroom/id/707508, August 22,2010 .

[204] G. Galen. What cloud computing really means. InfoWorld, [Online], Available: http://www.infoworld.com/d/cloudcomputing/what-cloud-computing-really-means-031, June 6, 2009.

[205] Cloud computing, [Online], Available: http://en. wikipedia.org/wiki/Cloud_computing, November 27, 2011.

[206] Cloud computing, [Online], Available: http://www.cloudcomputingdefined.com/, July 17 , 2010 .

[207] T. Segaran, J. Hammerbacher. Beautiful Data: The Stories Behind Elegant Data Solutions, Sebastopol: O'Reilly Media, 2009.

[208] T. White. Hadoop: The Definitive Guide, 3rd ed., Sebastopol: O'Reilly Media, 2012

[209] Y. Q. Xia. Cloud control systems. IEEE/CAA Journal of Automatica Sinica, vol. 2, no. 2, pp. 134-142, 2015.

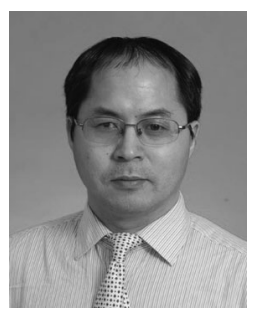

Yuan-Qing Xia received the M. Sc. degree in fundamental mathematics from Anhui University, China in 1998, and the $\mathrm{Ph}$. D. degree in control theory and control engineering from Beijing University of Aeronautics and Astronautics, China in 2001. From 2002 to 2003, he was a postdoctoral research associate in the Institute of Systems Science, Academy of Mathematics and System Sciences, Chinese Academy of Sciences China. From 2003 to 2006, he was with the National University of Singapore and the University of Glamorgan, UK, as a research fellow, respectively. From 2007 to 2008, he was a guest professor with Innsbruck Medical University, Austria. In 2012, he was appointed as Xu Teli Distinguished Professor at Beijing Institute of Technology and obtained the National Science Foundation for Distinguished Young Scholars of China. Since 2008, he has been working for Beijing Institute of Technology as a professor. He has published eight monographs in Springer and John Wiley, and more than 100 papers in journals. He obtained the Second 
Award of the Beijing Municipal Science and Technology (No.1) in 2010, the Second National Award for Science and Technology (No.2) in 2011, and the Second Natural Science Award of The Ministry of Education (No. 1) in 2012

His research interests include networked control systems, robust control, active disturbance rejection control and flight control.

E-mail: xia_yuanqing@bit.edu.cn (Corresponding author)

ORCID iD: 0000-0002-5977-4911

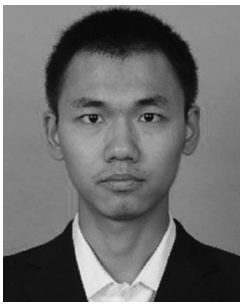

Yu-Long Gao received the B. Sc. degree in automation from Beijing Institute of Technology, China. He is now pursuing the M. Sc. degree in control science and engineering in Beijing Institute of Technology.

His research interests include networked control system, model predictive control, and multi-agent system.

E-mail: dfgaoyulong@gmail.com

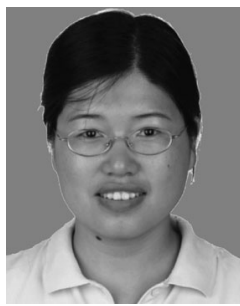

Li-Ping Yan received the B. Sc. degree and M.Sc. degree both in mathematics from Henan University, China in 2000 and 2003, respectively, and received the $\mathrm{Ph}$. D. degree in control science and engineering from Tsinghua University, China in 2007. From January 2007 to July 2009, she was a postdoctoral research associate in the Equipment Academy of Airforce, China. Since July 2009, she has been with the School of Automation,
Beijing Institute of Technology (BIT), China. From March 2012 to March 2013, supported by China Scholarship Council (CSC), she was a visiting scholar in the University of New Orleans, USA. Currently, she is an associate professor in BIT. She has co-authored two books and more than 40 journal and conference papers.

Her research interests include multisensor data fusion, state estimation, image registration, intelligent navigation, and integrated navigation.

E-mail: liping.yan@gmail.com

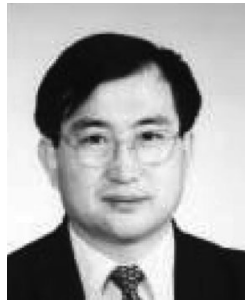

Meng-Yin Fu received the B. Sc. degree in radio electronics from Liaoning University, China in 1987, the M. Sc. degree in control theory from Beijing Institute of Technology, China in 1992, and the Ph. D. degree in geodetic engineering from Institute of Geodesy and Geophysics, Chinese Academy of Sciences, China in 2000. From August 1987 to September 1989, he worked as an assistant engineer at the Weather Bureau of Inner Mongolia. Since March 1992, he has been with Beijing Institute of Technology as a professor. He has coauthored three books and published more than 50 papers in technical journals and conferences. He is an executive director of the Chinese Society of Inertial Technology, fellow of Beijing Association for Science and Technology.

His research interests include inertial navigation and intelligent navigation, guidance and control.

E-mail: fumy@bit.edu.cn 